\title{
Telomere-dependent and telomere- independent roles of RAP1 in regulating human stem cell homeostasis
}

\author{
Xing Zhang ${ }^{1,2,6}$, Zunpeng Liu ${ }^{1,6}$, Xiaoqian Liu', Si Wang ${ }^{2,3,7}$, Yiyuan Zhang ${ }^{3,6}$, Xiaojuan $\mathrm{He}^{2}$, \\ Shuhui Sun ${ }^{3}$, Shuai Ma ${ }^{3}$, Ng Shyh-Chang ${ }^{1,6,7}$, Feng Liu ${ }^{5,6,7}$, Qiang Wang,6,7, Xiaoqun Wang ${ }^{3,6,7}$, Lin Liu ${ }^{4}$, \\ Weiqi Zhang, ${ }^{2,3,6,7 凶}$, Moshi Song, ${ }^{5,6,7 \bowtie}$, Guang-Hui Liu ${ }^{2,3,6,7,8,9 \bowtie}$, Jing Qu ${ }^{1,6,7 凶}$ \\ ${ }^{1}$ State Key Laboratory of Stem Cell and Reproductive Biology, Institute of Zoology, Chinese Academy of Sciences, \\ Beijing 100101, China \\ ${ }^{2}$ Advanced Innovation Center for Human Brain Protection, National Clinical Research Center for Geriatric Disorders, Xuanwu \\ Hospital Capital Medical University, Beijing 100053, China \\ ${ }^{3}$ National Laboratory of Biomacromolecules, CAS Center for Excellence in Biomacromolecules, Institute of Biophysics, \\ Chinese Academy of Sciences, Beijing 100101, China \\ ${ }^{4}$ State Key Laboratory of Medicinal Chemical Biology, Nankai University, Tianjin 300071, China \\ ${ }^{5}$ State Key Laboratory of Membrane Biology, Institute of Zoology, Chinese Academy of Sciences, Beijing 100101, China \\ ${ }^{6}$ University of Chinese Academy of Sciences, Beijing 100049, China \\ 7 Institute for Stem cell and Regeneration, Chinese Academy of Sciences, Beijing 100101, China \\ ${ }^{8}$ Key Laboratory of Regenerative Medicine of Ministry of Education, Institute of Aging and Regenerative Medicine, Jinan \\ University, Guangzhou 510632, China \\ ${ }^{9}$ Beijing Institute for Brain Disorders, Capital Medical University, Beijing 100069, China \\ $\bowtie$ Correspondence: weiqizhang@aliyun.com (W. Zhang), songmoshi@ioz.ac.cn (M. Song), ghliu@ibp.ac.cn (G.-H. Liu), \\ qujing@ioz.ac.cn (J. Qu)
}

Received December 2, 2018 Accepted January 3, 2019

\begin{abstract}
RAP1 is a well-known telomere-binding protein, but its functions in human stem cells have remained unclear. Here we generated RAP1-deficient human embryonic stem cells (hESCs) by using CRISPR/Cas9 technique and obtained RAP1-deficient human mesenchymal stem cells (hMSCs) and neural stem cells (hNSCs) via directed differentiation. In both hMSCs and hNSCs, RAP1 not only negatively regulated telomere length but also acted as a transcriptional regulator of RELN by tuning the methylation status of its gene promoter. RAP1 deficiency enhanced self-renewal and delayed senescence in hMSCs, but not in hNSCs, suggesting complicated lineage-specific effects of RAP1 in adult stem cells.
\end{abstract}

Xing Zhang and Zunpeng Liu have contributed equally to this work.

Electronic supplementary material The online version of this article (https://doi.org/10.1007/s13238-019-0610-7) contains supplementary material, which is available to authorized users.
Altogether, these results demonstrate for the first time that RAP1 plays both telomeric and nontelomeric roles in regulating human stem cell homeostasis.

KEYWORDS RAP1, stem cell, telomere, RELN, methylation

\section{INTRODUCTION}

Telomeres, the short repeat sequences at the chromosome ends in eukaryotic organisms (Greider, 1991; Blackburn, 2001), are indispensable for the maintenance of genome stability (Londono-Vallejo, 2004; Zhang et al., 2018). Telomere erosion induces cellular senescence in human cells (Proctor and Kirkwood, 2003; Lopez-Otin et al., 2013; Xu et al., 2013; Blackburn et al., 2015; Bourgeron et al., 2015; Zhu et al., 2018). Shelterin, also known as telosome, is a protein complex recruited by the telomeres. Shelterin is involved in the maintenance of advanced telomere structures and regulates the telomere niche via interaction with numerous protein components (Palm and de Lange, 2008; 
Xin et al., 2008; Bandaria et al., 2016). Human shelterin is composed of six components: RAP1, TRF1, TRF2, TIN2, TPP1 and POT1 (Schmutz and de Lange, 2016).

RAP1 (repressor/activator protein 1), also known as TERF2IP (TERF2 interaction protein), was first reported as a transcriptional regulator in Saccharomyces cerevisiae (Shore and Nasmyth, 1987). RAP1 is an evolutionarily conserved protein (Khurana et al., 2013; Kabir et al., 2014) that contains BRCT, Myb and C-terminal protein interaction domains (Kabir et al., 2010). RAP1 regulates telomeres by directly binding to double-stranded telomeric DNA (budding yeast) or interacting with a group of homologs consisting of Taz1 (fission yeast), TRF (trypanosome), TRFA (zebrafish) or TRF2 (mammals) (Kyrion et al., 1993; Kanoh and Ishikawa, 2001; Yang et al., 2009; Wagner et al., 2017). In yeast, RAP1 is implicated in the regulation of telomeric heterochromatin status by recruiting Sir $/ 3 / 4$ protein complex (Moretti and Shore, 2001; Doerks et al., 2002); RAP1 deficiency leads to excessive telomere extension (Luo et al., 2002). However, the role of mammalian RAP1 is controversial. RAP1 deficiency results in shortened telomeres only in certain mouse tissues (Martinez et al., 2010, 2016). Similarly, in immortalized human cell lines, its deficiency causes telomere elongation in some cases, but exerts no effect on telomere length in other cases ( $\mathrm{Li}$ and de Lange, 2003; O'Connor et al., 2004; Kabir et al., 2014; Kim et al., 2017). In addition to the role in regulating telomere length, RAP1 has also been reported to suppress the expression of telomeric repeat-containing RNA (TERRA) and subtelomeric genes (Nanavaty et al., 2017). Recently, emerging evidences have suggested that mammalian RAP1 may also play a nontelomeric role by occupying specific extratelomeric DNA regions as a transcriptional factor and regulating gene expression (Martinez et al., 2010, 2013, 2016; Yang et al., 2011). However, the underlying molecular mechanisms remain to be elucidated.

Senescence or exhaustion of adult stem cell pools is considered as a hallmark of aging (Liu et al., 2011, 2014; Lopez-Otin et al., 2013; Goodell and Rando, 2015; Zhang et al., 2015; Pan et al., 2016; Ren et al., 2017b; Yang et al., 2017; Wang et al., 2018b; Wu et al., 2018). In the search for therapeutic modalities to revitalize adult stem cells, telomere extension has attracted attention, but there was a lack of safe strategies and further validation. In this study, we found that RAP1 regulated human stem cell senescence in both telomere-dependent and telomere-independent manners. We knocked out RAP1 in hESCs by the CRISPR/Cas9 technique and differentiated RAP1-deficient hESCs into two different types of human adult stem cells, hMSCs and hNSCs. RAP1 deficiency was sufficient for telomere extension in both hMSCs and hNSCs, but delayed senescence only in hMSCs. We further identified that RELN was silenced with promoter hypermethylation in RAP1-deficient cells and that the RAP1-RELN pathway partially contributed to the regulation of senescence in hMSCs.
Figure 1. Generation and characterization of $\mathrm{RAP}^{-1-}$ hESCs. (A) Schematic representation of the deletion of the exon 2 of RAP1 in hESCs via CRISPR/Cas9-facilitated $H R$. The green triangles represented FRT sites and the red cross demonstrated the region of sgRNA. (B) Schematic representation of the primers used for genomic $P C R$ and qRT-PCR to confirm RAP1 knockout. (C) Genomic PCR analysis demonstrated that the exon 2 of RAP1 was deleted from the genome. LA and RA represented left and right homology arm, respectively. (D) qRT-PCR analysis demonstrated the deletion of RAP1 at the transcriptional level in $\mathrm{RAP}^{-1-} \mathrm{hESC}$ by primers $\mathrm{P} 8$ and P9. Data were presented as the mean $\pm \mathrm{SEM}, n=3 .{ }^{* * *} P<0.001$. (E) Western blotting analysis verified the absence of RAP1 in $R A P 1^{-1-}$ hESCs. $\beta$-Actin was used as a loading control. (F) RT-PCR for pluripotency markers demonstrated comparable expression in WT and $R A P 1^{-1-}$ hESCs. $18 \mathrm{~S}$ rRNA was used as a loading control. (G) Representative brightfield and immunofluorescence micrographs of WT and $R A P 1^{-1-}$ hESCs showed normal morphology of hESCs and comparable expression of pluripotency markers, respectively. Scale bar, $50 \mu \mathrm{m}$. (H) DNA methylation status of the OCT4 promoter in WT and $R A P 1^{-/-}$hESCs showed normal hypomethylation. (I) Immunostaining of representative markers of the three germ layers in teratomas

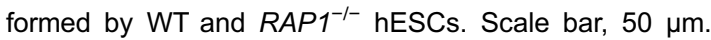
(J) Clonal expansion analysis of WT and $R A P 1^{-1-} \mathrm{hESCs}$. Data were presented as the mean $\pm \mathrm{SEM}, n=3$. NS, not significant. (K) Immunostaining of the proliferation marker Ki67 in WT and $R A P 1^{-1-}$ hESCs. Scale bar, $50 \mu \mathrm{m}$. Data were presented as the mean \pm SEM, $n=6$. NS, not significant. (L) Cell cycle analysis of WT and $R A P 1^{-1-}$ hESCs. Data were presented as the mean \pm SEM, $n=3$. NS, not significant. (M) Karyotype analysis of $R A P 1^{-1-}$ hESCs showed normal karyotype. $n=10$.

\section{RESULTS}

\section{RAP1-deficient hESCs maintained pluripotency}

To study the biological functions of human RAP1, we generated RAP1-knockout hESCs by deleting the exon 2 of RAP1 (Kabir et al., 2014) via CRISPR/Cas9-facilitated homologous recombination (HR) (Wang et al., 2018a, b) (Fig. 1A). Biallelic deletion of the exon 2 of RAP1 was confirmed by genomic PCR (Fig. $1 \mathrm{~B}$ and $1 \mathrm{C}$ ). Moreover, the successful ablation of RAP1 mRNA and protein was validated by quantitative reverse transcription PCR (qRT-PCR) and Western blotting (Fig. 1D and 1E).

$R A P 1^{-1-}$ hESCs exhibited normal colony morphology, expressed the pluripotency markers OCT4, SOX2 and NANOG (Fig. 1F and 1G) and maintained hypomethylation at the OCT4 promoter (Fig. $1 \mathrm{H}$ ). Meanwhile, teratoma analysis proved that $R A P 1^{-1-}$ hESCs were able to differentiate into endoderm, mesoderm and ectoderm lineages in vivo (Fig. 1I). Normal proliferation ability was verified via clonal 


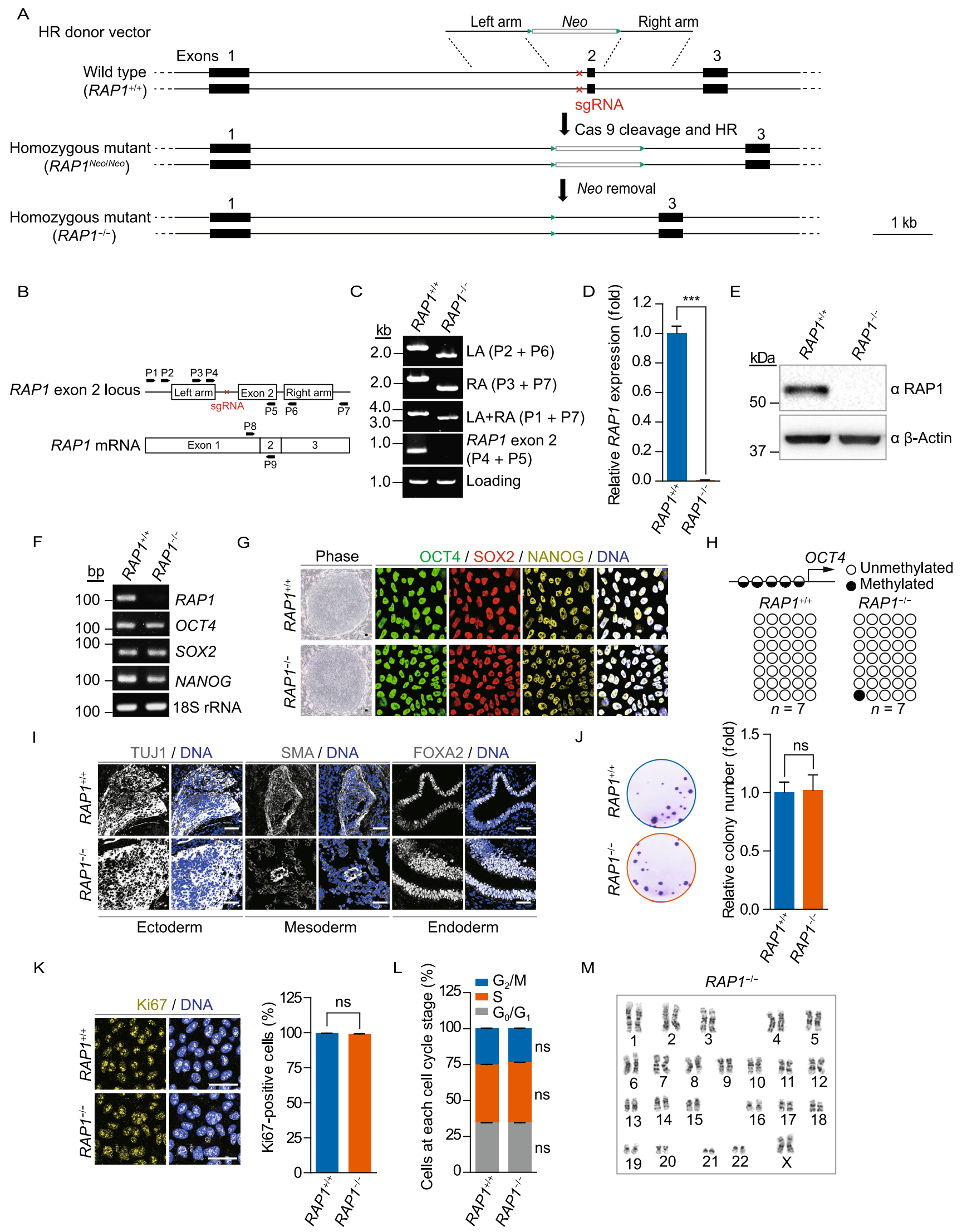


expansion assay, Ki67 immunostaining, and cell cycle analysis (Fig. $1 \mathrm{~J}-\mathrm{L}$ ). In addition, $R A P 1^{-1-} \mathrm{hESCs}$ maintained normal karyotype (Fig. 1M). Taken together, these results indicate that $\mathrm{RAP}^{-1-}$ hESCs maintained normal pluripotency and self-renewal capability.

\section{RAP1 deficiency delayed hMSC senescence}

To elucidate the role of RAP1 in human somatic stem cells, we first differentiated wild type (WT) and $R A P 1^{-/-} \mathrm{hESCs}$ into hMSCs. Compared to WT hMSCs, RAP1 ${ }^{-1-}$ hMSCs exhibited normal morphology and expressed hMSC-specific surface markers including CD73, CD90 and CD105 (Fig. 2A and 2B). The absence of RAP1 mRNA and protein was confirmed by qRT-PCR, immunofluorescence and Western blotting (Fig. 2C-E). In addition, $R A P 1^{-1-}$ hMSCs maintained the ability to differentiate into chondrocytes, adipocytes and osteoblasts (Fig. 2F).

Higher proliferation ability of $R A P 1^{-1-}$ hMSCs was observed through serial passaging relative to that of WT hMSCs, in which growth arrest occurred at passage 9; by

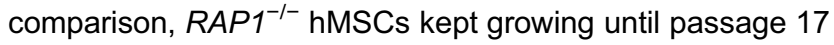
(Fig. 2G). Ki67 immunostaining and clonal expansion formation assays further confirmed improved proliferation ability of $R A P 1^{-1-} \mathrm{hMSCs}$ (Fig. $2 \mathrm{H}$ and 2l). Consistently, $R A P 1^{-1-}$ hMSCs had more cells in $S$ phase relative to WT

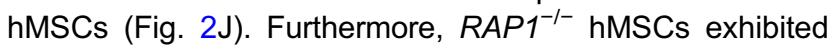
lower rate of senescence-associated $\beta$-galactosidase (SA- $\beta$ gal)-positive cells and less abundant senescence-associated proteins at late passage (Fig. $2 \mathrm{~K}$ and $2 \mathrm{~L}$ ). In line with improved proliferation and delayed senescence, $R A P 1^{-1-}$ hMSCs were resistant to in vivo attrition after being implanted into the tibialis anterior (TA) muscle of nude mice (Fig. 2M). Genome-wide copy number variation (CNV) analysis demonstrated high genomic integrity in $R A P 1^{-1-}$ hMSCs (Fig. 2N), excluding the possibility that the cellular phenotypes resulted from chromosomal aberrations. Taken together, these results indicate that RAP1 deficiency promoted proliferation and slowed senescence in hMSCs.

\section{RAP1 deficiency led to telomere elongation in hMSCs}

Given the known role of RAP1 as a telomere binding protein, we next evaluated telomeric alterations in $R A P 1^{-1-} \mathrm{hMSCs}$.

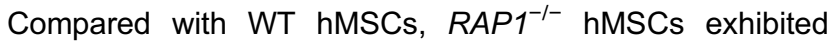
longer telomeres, which was verified by multiple methods, including Southern blotting, flow FISH (flow cytometry and FISH) and genomic qPCR (Fig. 3A-C). The elongated telomeres were partially rescued by the re-introduction of RAP1 in RAP1 ${ }^{-/-}$hMSCs (Fig. 3D). A chromatin immunoprecipitation (ChIP)-PCR assay further demonstrated the association of RAP1 with the telomeres in WT hMSCs, rather than in RAP1-deficient hMSCs (Fig. 3E and 3F). Meanwhile, RAP1 deficiency resulted in less enrichment of H3K9me2, a heterochromatin marker, at the telomeres in hMSCs (Fig. 3G). Consistent with a previous observation that the
Figure 2. $\mathrm{RAP}^{-1-} \mathrm{hMSC}$ exhibited retarded cellular senescence. (A) Brightfield micrographs of hMSCs showed normal morphology. Scale bar, $50 \mu \mathrm{m}$. (B) Flow cytometry demonstrated that sorted hMSCs uniformly expressed the MSC-specific surface markers CD73, CD90 and CD105. (C) qRT-PCR analysis demonstrated the deletion of RAP1 at the transcriptional level in $R A P 1^{-/}$ hMSCs by primers P8 and P9. Data were presented as the mean \pm SEM, $n=3$. ${ }^{* *} P<0.001$. (D) Immunofluorescence micrographs of RAP1 in WT and RAP $1^{-/-}$hMSCs. Scale bar, $10 \mu \mathrm{m}$. (E) Western blotting analysis demonstrated the absence of RAP1 in RAP1 ${ }^{-/-}$hMSCs. $\beta$-Actin was used as a loading control. ( $F$ ) Characterization of the differentiation potential of hMSCs. Toluidine blue O, Oil Red O and Von Kossa staining were used to detect chondrocytes, adipocytes and osteoblasts, respectively. Scale bar, $200 \mu \mathrm{m}$. (G) Cell growth curves showed the enhanced proliferation ability of $R A P 1^{-1-}$ hMSCs. $(\mathrm{H})$ Immunostaining of the proliferation marker Ki67 in WT and $R A P 1^{-/-}$hMSCs. EP (early passage) and LP (late passage) represented $\mathrm{P} 2$ and $\mathrm{P9}$, respectively. Scale bar, $50 \mu \mathrm{m}$. Data were presented as the mean \pm SEM, $n=6$. NS, not significant, ${ }^{* \star *} P<0.001$. (I) Clonal expansion analysis of WT and $R A P 1^{-1-} \mathrm{hMSCs}$. EP and LP represented $\mathrm{P} 2$ and $\mathrm{P} 9$, respectively. Scale bar, $50 \mu \mathrm{m}$. Data were presented as the mean \pm SEM, $n=3$. ${ }^{\star *} P<0.01,{ }^{* * *} P<0.001$. (J) Cell cycle analysis of WT and $R A P 1^{-1-}$ hMSCs (LP). Data were presented as the mean \pm SEM, $n=3$. NS, not significant, ${ }^{*} P<0.05$, ${ }^{* *} P<0.001$. (K) SA- $\beta$-gal staining of WT and $R A P 1^{-1-}$ hMSCs. EP and LP represented $P 2$ and $P 9$, respectively. Scale bar, $50 \mu \mathrm{m}$. Data were presented as the mean $\pm \mathrm{SEM}, n=6$. NS, not significant, ${ }^{* * *} P<0.001$. (L) Western blotting analysis showed decreased expression of P16 and P21 in RAP1 ${ }^{-1-}$ hMSCs (LP). B-Actin was used as a loading control. (M) Photon flux from TA muscle implanted with WT (left) and $R A P 1^{-1-}$ (right) hMSCs (LP) expressing luciferase. Data were presented as the mean \pm SEM of $R A P 1^{-1-}$ to WT ratios $\left(\log _{2}\right.$ (fold change)), $n=5 .{ }^{* *} P<0.01,{ }^{* * *} P<0.001$. (N) Identification of CNVs by whole-genome sequencing analysis in WT and $R A P 1^{-1-} \mathrm{hMSCs}$ (EP) showed genomic integrity in $R A P 1^{-1-}$ hMSCs.

heterochromatin status of telomeres affects TERRA expression (Arnoult et al., 2012), TERRA transcripts were upregulated in $\mathrm{RAP1}^{-/-} \mathrm{hMSCs}$ (Fig. 3H), which was reversible upon addition of exogenous RAP1 (Fig. 3I). However, RAP1-deficient hMSCs exhibited no effect on the resistance to kinds of DNA damage stressors (Fig. S1A and S1B). Taken together, these results suggest that RAP1 counteracted telomere length in hMSCs.

\section{RAP1 deficiency downregulated RELN in hMSCs}

To investigate whether any telomere-independent function of RAP1 was present, we carried out genome-wide RNA 
A

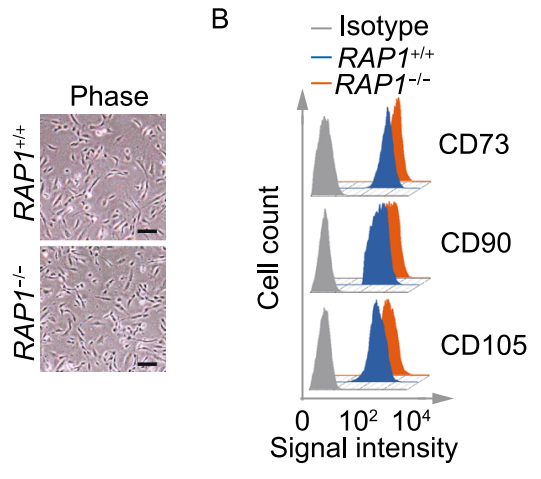

F

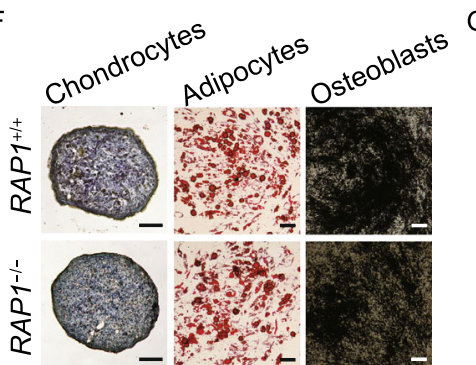

$G \stackrel{\text { g }}{\Xi}$
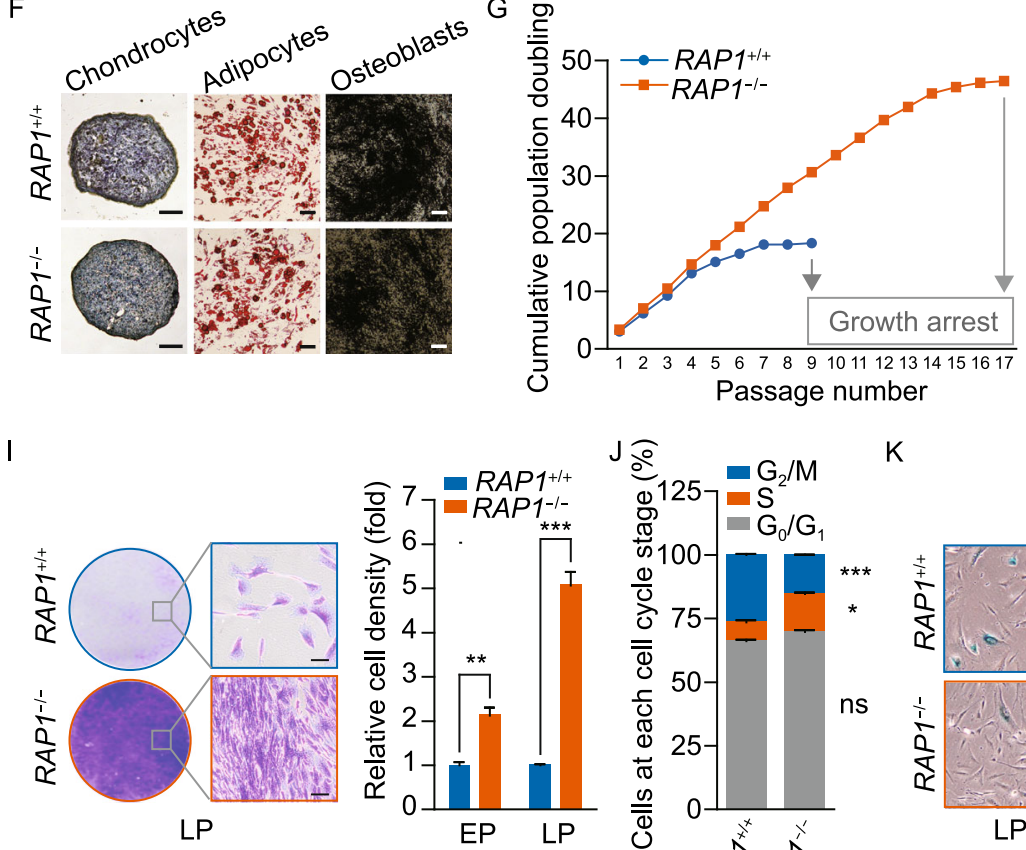

M

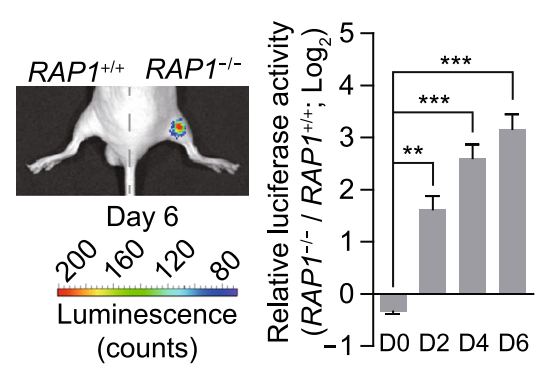

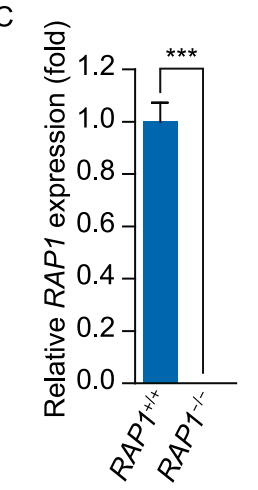

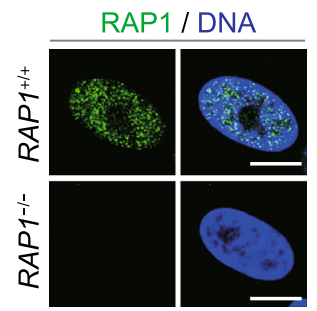

$\mathrm{H}$
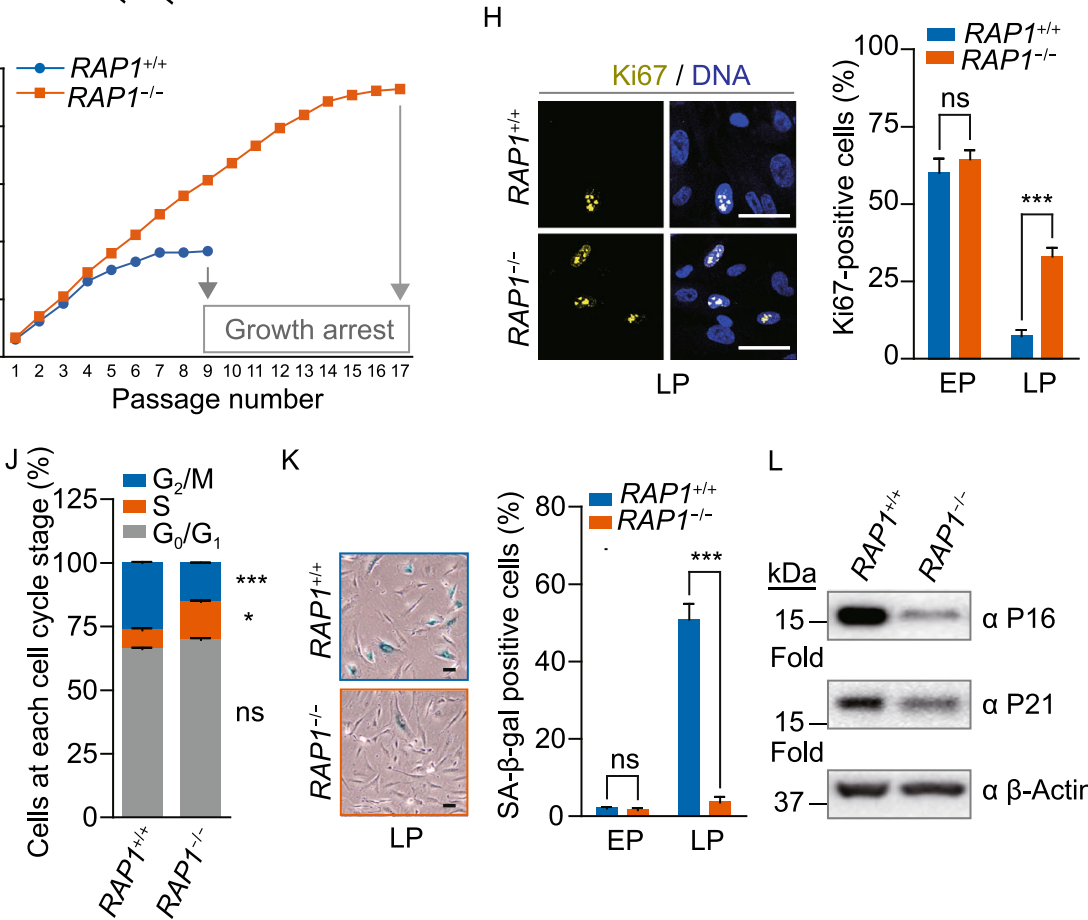

K
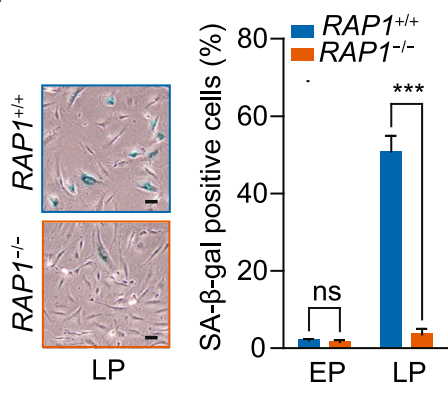

L

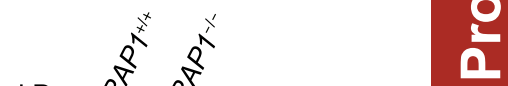

$\mathrm{N}$

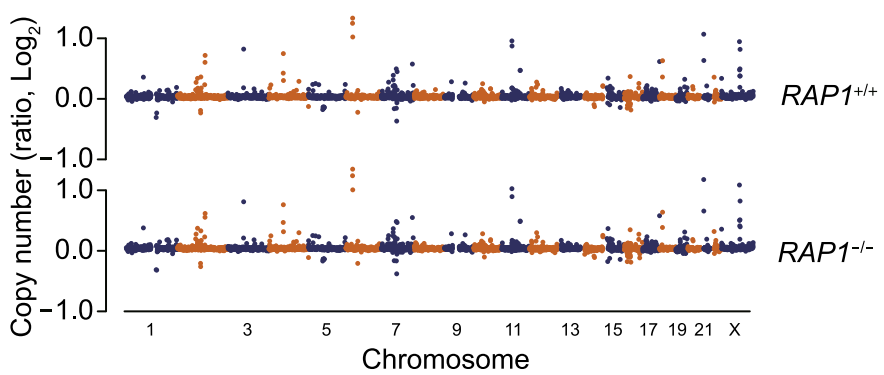


A

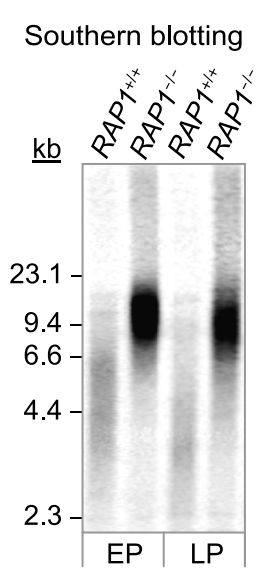

B

Flow FISH

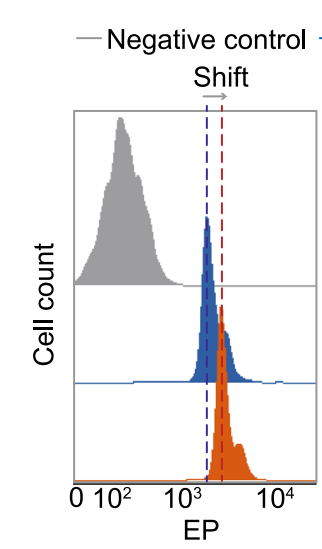

C

qPCR
D qPCR
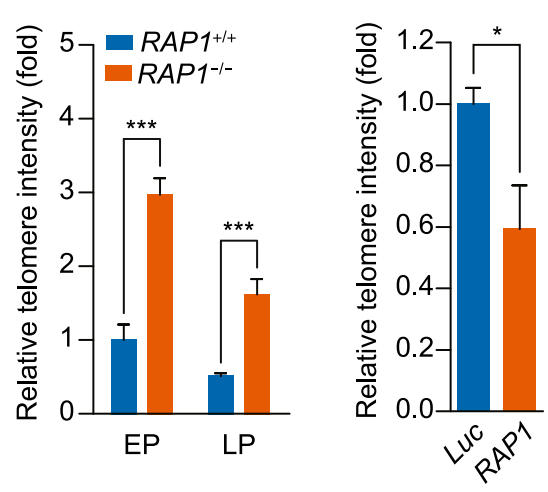

E

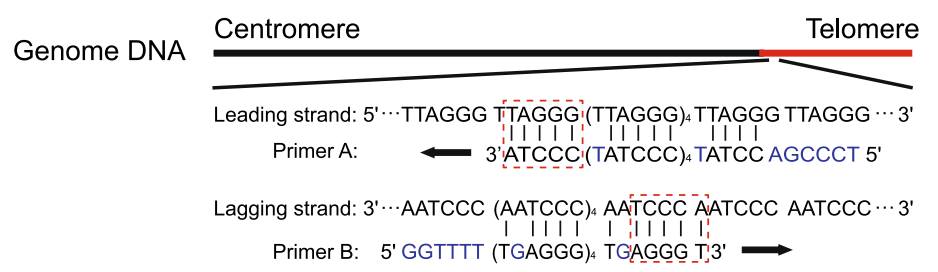

Primer A:

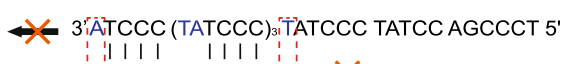

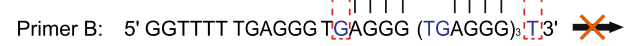

$\mathrm{H}$

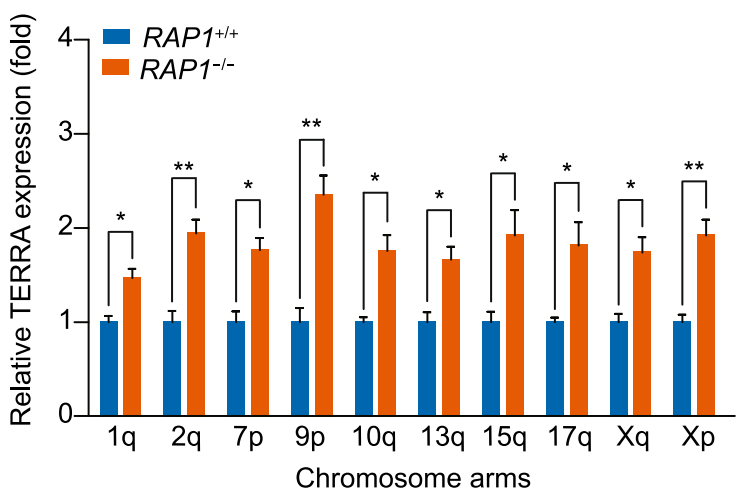

$\mathrm{F}$

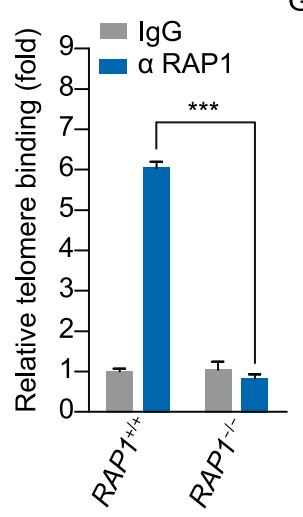

G

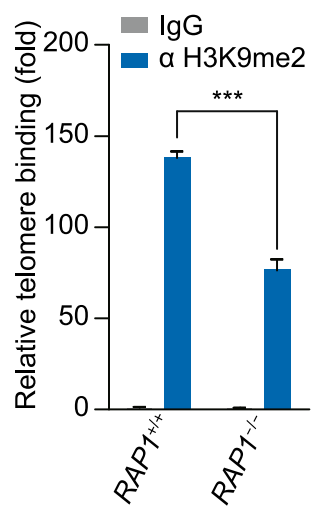

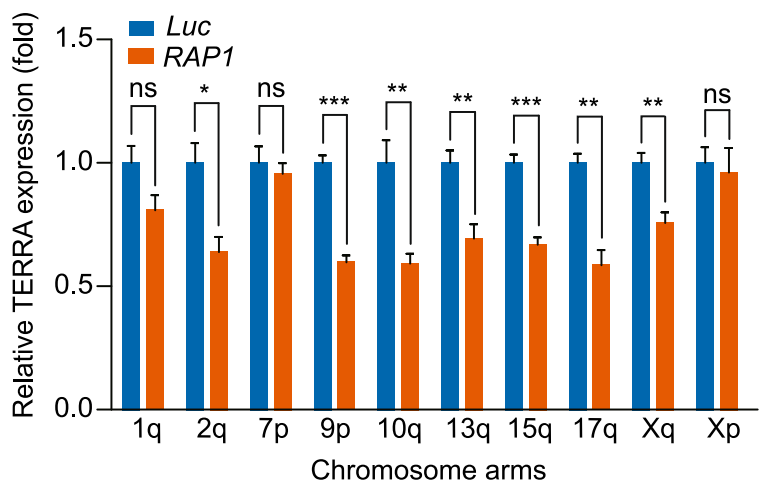

Figure 3. RAP1 ${ }^{-1-} \mathrm{hMSCs}$ contained longer telomeres. (A) Terminal restriction fragment analysis of WT and $R A P 1^{-1-} \mathrm{hMSCs}$ by Southern blotting demonstrated longer telomeres in $R A P 1^{-1-} \mathrm{hMSCs}$. EP and LP represented P2 and P9, respectively. (B) Telomere

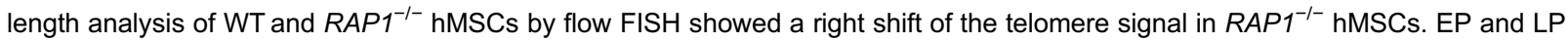
represented P2 and P9, respectively. (C) Telomere length analysis of WT and RAP1 ${ }^{-1-}$ hMSCs by qPCR. EP and LP represented P2 and P9, respectively. Data were presented as the mean $\pm \mathrm{SEM}, n=3 .{ }^{* * *} P<0.001$. (D) Telomere length analysis of $R A P 1^{1--} \mathrm{hMSCs}$ expressing luciferase (LuC) or RAP1 by $\mathrm{qPCR}$. Data were presented as the mean $\pm \mathrm{SEM}, n=3$. ${ }^{*} P<0.05$. (E) Schematic representation of the primers designed for telomere detection explaining how primer $A$ and $B$ specifically amplified telomeres rather than forming primer dimers. (F) ChIP-PCR analysis of RAP1 enrichment at the telomeres in WT and RAP1 ${ }^{-1-} \mathrm{hMSCs}$ (EP). Data were presented as mean $\pm \mathrm{SEM}, n=3$. ${ }^{* *} P<0.001$. (G) ChIP-PCR analysis of H3K9me2 enrichment at the telomeres in WT and $R A P 1^{-/-}$ hMSCs (EP) indicated a looser telomere structure in RAP1 ${ }^{-/-}$hMSCs. Data were presented as the mean \pm SEM, $n=3 .{ }^{* * *} P<0.001$. (H) qRT-PCR analysis showed that RAP1 ${ }^{-1-}$ hMSCs (EP) expressed more TERRA. Data were presented as the mean $\pm \mathrm{SEM}, n=3$. NS, not significant, ${ }^{*} P<0.05,{ }^{* *} P<0.01$. (I) TERRA analysis of $R A P 1^{-1-}$ hMSCs expressing luciferase or RAP1 by qRT-PCR. Data were presented as the mean \pm SEM, $n=3$. NS, not significant, ${ }^{*} P<0.05,{ }^{* *} P<0.01,{ }^{* \star} P<0.001$. 
sequencing (RNA-seq) analysis in WT and $R A P 1^{-/-}$hMSCs. In $R A P 1^{-1-} \mathrm{hMSCs}$, there were 134 downregulated genes and 152 upregulated genes comparing to those in WT hMSCs $\left(P\right.$ adj $<0.05$ and $\mid \log _{2}$ (fold change) $\mid>0.5$ ) (Tables S1 and S2). Biological process gene ontology (GO-BP) enrichment analysis revealed that the upregulated genes were mainly associated with development and cellular differentiation, and the downregulated genes with cell adhesion and extracellular matrix organization (Fig. 4A). In addition, known RAP1 target genes in mammals were mostly unaffected in RAP1-deficient hMSCs (Martinez et al., 2010, 2013; Yang et al., 2011; Kabir et al., 2014) (Fig. 4B). Notably, we found that $R E L N$, a negative regulator of proliferation (Sato et al., 2006; Kundakovic et al., 2007; Schulze et al., 2017), was dramatically downregulated in RAP1-deficient hMSCs (Fig. 4C-F). Furthermore, the expression level of RELN was partially rescued by exogenously expressed RAP1 (Fig. 4G). These data suggest that RAP1 positively regulated RELN expression in hMSCs.

We further found that RAP1 was associated with the RELN promoter by ChIP analysis (Fig. $4 \mathrm{H}$ ). Considering that the transcription of RELN depends on the methylation status of its promoter (Sato et al., 2006; Kundakovic et al., 2007, 2009; Lintas et al., 2016; Nabil Fikri et al., 2017), we next evaluated changes in the methylation status of the RELN promoter upon RAP1 deletion. In line with decreased RELN expression, RELN promoter was hypermethylated in $R A P 1^{-/-} \mathrm{hMSCs}$ (Fig. 4I). These data suggest that the binding of RAP1 to RELN promoter in WT hMSCs was associated with a lower methylation level at this region, which may facilitate the transcription of RELN. To determine whether downregulation of RELN partially contributed to improved proliferation in RAP1-deficient hMSCs, we knocked down RELN via a lentiviral shRNA vector (Fig. 4J). Downregulation of RELN promoted proliferation ability and delayed senescence in WT hMSCs (Fig. 4K$\mathrm{N})$. Taken together, these data indicate that RAP1 regulated the proliferation and senescence of hMSCs at least in part via the epigenetic derepression of RELN expression.

Since RELN encodes Reelin, which is a typical marker of preplate/Cajal-retzius cells in the brain and plays an important role in the development of the nervous system (Lancaster et al., 2013; Lancaster and Knoblich, 2014; Sekine et al., 2014; Ishii et al., 2016), we performed cerebral differentiation using a 3D culture based procedure (Fig. S2A) and achieved organoid-like cerebral structures by using WT and $R A P 1^{-1-} \mathrm{hESCs}$. As expected, the resultant organoidlike cerebral structures by $R A P 1^{-1-}$ hESCs lacked RAP1 expression and the layer containing preplate/Cajalretzius cells marked by Reelin (Fig. S2B-E), further supporting a role of RAP1 in regulating RELN expression.

\section{RAP1 had no effect on the proliferation and senescence of hNSCs}

To test whether RAP1 regulated cell proliferation and senescence in a cell type-specific manner, we differentiated
WT and $R A P 1^{-1-}$ hESCs into hNSCs. $R A P 1^{-1-}$ hNSCs demonstrated normal neural progenitor morphology, expressed the NSC-specific markers PAX6, SOX2 and Nestin (Fig. 5A), and maintained neuronal differentiation ability (Fig. S3A). Ablation of RAP1 protein in RAP $1^{1-}$ hNSCs was verified by immunofluorescence and Western blotting (Fig. 5B and 5C). RNA-seq analysis revealed a total of 124 downregulated genes and 68 upregulated genes in $R A P 1^{-1-}$ hNSCs comparing to those in WT hNSCs ( $P$ adj < 0.05 and $\mid \log _{2}($ fold change) $\mid>0.5$ ) (Tables S3 and S4). However, among the 192 differentially expressed genes between $R A P 1^{-1-}$ and WT hNSCs and 286 differentially expressed genes between $R A P 1^{-1-}$ and WT hMSCs, only 20 genes were overlapped (Fig. 5D). Notably, RELN was still one of the most downregulated genes in $R A P 1^{-/-}$hNSCs (Fig. 5E-H). Consistently, silencing of RELN expression in $R A P 1^{-1-} \mathrm{hNSC}$ s was associated with the hypermethylation at the RELN promoter (Fig. $5 \mathrm{I}$ and $5 \mathrm{~J}$ ). Similar to RAP1deficient hMSCs, depletion of RAP1 in hNSCs resulted in longer telomeres (Fig. 5K-M). However, no proliferation-

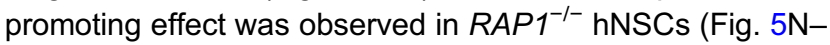
$\mathrm{Q}$ ). Additionally, the in vitro migration ability of hNSCs was also comparable between WT and $R A P 1^{-1-} \mathrm{hNSCs}$ (Fig. S3B). Therefore, RAP1 deficiency itself was insufficient to promote proliferation in hNSCs despite its marked effects on $R E L N$ expression and telomere length.

\section{DISCUSSION}

With the aid of the CRISPR/Cas9-mediated gene-editing technique, our study revealed for the first time that RAP1 negatively regulated telomere length as a telomere-binding protein and positively regulated the expression of RELN as a potential epigenetic regulator in both hMSCs and hNSCs. Interestingly, RAP1 functioned as a proliferation/senescence regulator only in hMSCs (Fig. 6), but not in hNSCs. Thus, our results provide an important evidence that RAP1 may play a role in regulating human stem cell homeostasis in a lineagespecific manner.

The effects of RAP1 in regulating cell proliferation have been controversial (Table S5). RAP1-knockout mice exhibit normal growth and lifespan (Martinez et al., 2010, 2013, 2016; Sfeir et al., 2010). However, RAP1 deficiency decreased the proliferation of the human hepatocellular carcinoma cell line HepG2 and non-small cell lung cancer cell line A549 (Zha et al., 2014; Xiao et al., 2017), but not that of the human fibrosarcoma cell line HT1080 (Kabir et al., 2014) or mouse MSCs (Ding et al., 2018). It is therefore likely that the effect of RAP1 in growth regulation is species or cell type-specific. In this study, we generated RAP1-knockout hESCs and obtained their hMSC and hNSC derivatives via directed differentiation. With these cell models, we observed a lineage-specific effect of RAP1 in regulating stem cell senescence despite the altered telomere length and RELN expression. It is possible that specific factors in RAP1knockout hNSCs may compensate for the effect of RAP1 


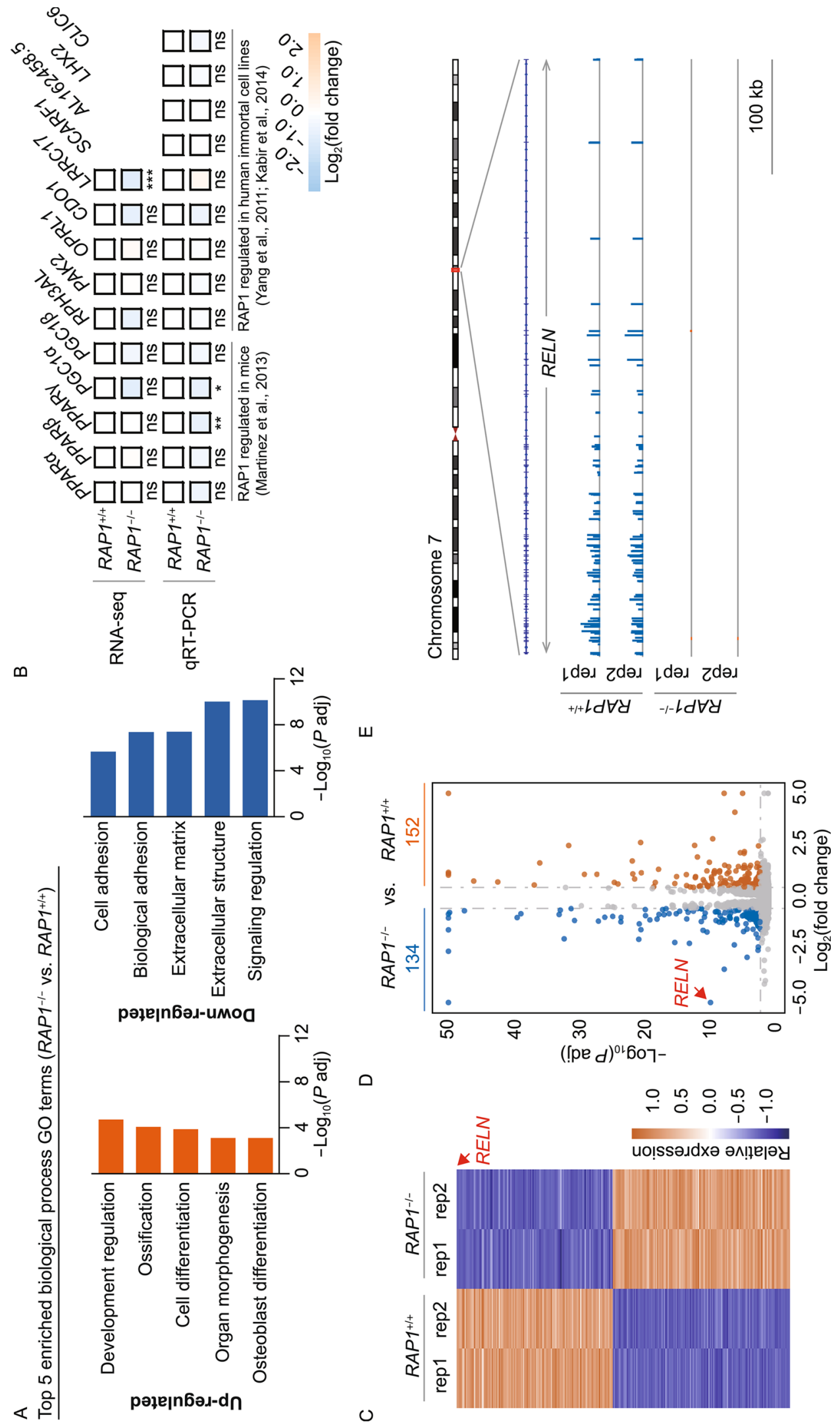


Figure 4. RAP1 regulated the expression of RELN in hMSCs. (A) Biological process GO enrichment analysis of differentially expressed genes in $R A P 1^{-/-}$vs. WT hMSCs (EP). The top 5 enriched biological process GO terms were shown. (B) Heatmap of reported genes regulated by RAP1 in mice or human immortalized cell lines. Relative expression of indicated genes tested by RNA-seq and qRT-PCR demonstrated comparable levels in WT and RAP1 ${ }^{-/-}$hMSCs (EP). $n=3$ (qRTPCR) or 2 (RNA-seq). NS, not significant, ${ }^{*} P$ (qRT-PCR) or $P$ adj (RNA-seq) $<0.05,{ }^{* \star} P$ or $P$ adj $<0.01,{ }^{* \star *} P$ or $P$ adj $<0.001$. (C) Heatmap of differentially expressed genes in $R A P 1^{-1-}$ vs. WT hMSCs (EP). RELN was labeled by an arrow. (D) Volcano plot of differentially expressed genes in $R A P 1^{-1-}$ vs. WT hMSCs (EP). RELN was labeled by an arrow. (E) Transcriptional signals of $R E L N$ in $R A P 1^{-1-}$ vs. WT hMSCs (EP). Transcriptional signals were normalized by RPKM at a bin size of $10 \mathrm{bp}$. (F) qRT-PCR analysis verified that RELN was downregulated in $R A P 1^{-1-}$ hMSCs (EP). Data were presented as the mean \pm SEM, $n=3$. ${ }^{* * \star} P<0.001$. (G) qRT-PCR analysis demonstrated that expressing RAP1 partially rescued the expression of RELN in $R A P 1^{-1-}$ hMSCs. Data were presented as the mean \pm SEM, $n=3$. ${ }^{*} P<0.05$. (H) Diagram showing that RAP1 bound immediately upstream of the TSS of RELN in WT hMSCs (EP). ChIP-PCR analysis showed that RAP1 was enriched at the RELN promoter region. Data were presented as the mean $\pm \mathrm{SEM}, n=3$. ${ }^{* *} P<0.01$. (I) Methylation-specific PCR analysis of the RELN promoter in WT and $R A P 1^{-1-} \mathrm{hMSCs}$ (EP) demonstrated hypermethylation of the RELN promoter in RAP1 ${ }^{-1-}$ hMSCs. Data were presented as the mean \pm SEM, $n=3 .{ }^{* *} P<0.01$. (J) qRT-PCR analysis demonstrated that RELN shRNA (sh $R E L N$ ) effectively decreased the mRNA of RELN than control shRNA (sh CTRL). Data were presented as the mean \pm SEM, $n=3$. ${ }^{* *} P<0.01$. (K) Cell growth curves showed that reducing the expression of RELN enhanced the proliferation ability of WT hMSCs. (L) Immunostaining of the proliferation marker Ki67 in WT hMSCs transfected with control or RELN shRNA. Scale bar, $50 \mu \mathrm{m}$. Data were presented as the mean \pm SEM, $n=6$. ${ }^{* * *} P<0.001$. (M) Clonal expansion analysis of WT hMSCs transfected with control or RELN shRNA. Scale bar, $50 \mu \mathrm{m}$. Data were presented as the mean $\pm \mathrm{SEM}, n=3 .{ }^{* \star} P<0.01$. (N) SA- $\beta$ gal staining of WT hMSCs transfected with control or RELN shRNA. Scale bar, $50 \mu \mathrm{m}$. Data were presented as the mean \pm SEM, $n=6 .{ }^{* * *} P<0.001$.

deficiency on proliferation regulation. The cell type-specific effects could also be explained by the notion that RAP1 regulates distinct sets of genes in hMSCs and hNSCs. For instance, among the most upregulated genes in $R A P 1^{-1-}$ hMSCs, several are implicated in cell proliferation, such as S100A6 (Donato et al., 2017), PLXNA4 (Di Palma et al., 2013), MAGED4B (Ferrara-Romeo et al., 2018) and PAX8 (Di Palma et al., 2013). However, none of those gene expression changes were observed in $R A P 1^{-/-}$hNSCs.

Although RAP1 is a well-known telomere-binding protein, the mechanism by which RAP1 regulates telomere length remains controversial. RAP1 has been reported to protect against telomere attrition in tail skin and small intestine in mice (Martinez et al., 2010, 2016), but negatively regulates telomere length in human immortalized cells ( $\mathrm{Li}$ and de Lange, 2003; O'Connor et al., 2004; Kim et al., 2017). By contrast, Kabir et al. knocked out the exon 2 of RAP1 by TALEN technique in multiple human immortalized cell lines and observed no effect of RAP1 deficiency on telomere length or any other telomere-related parameters (Kabir et al., 2014). Given that the telomere biology of rodent cells and human immortalized cell lines is substantially different from that of human diploid cells (Calado and Dumitriu, 2013; Reddel, 2014), it is necessary to clarify the role of RAP1 in human diploid cells in a physiological context. Therefore, the RAP1-deficient human diploid cells that we established here represent superior models for gaining a better understanding of RAP1-mediated telomere regulation in human adult stem cells. We observed that RAP1 deficiency elongated telomeres in both hMSCs and hNSCs, which is consistent with observations in lower eukaryotes (Kanoh and Ishikawa, 2001) and the "shelterin counting mechanism" theoretical model (Smogorzewska and de Lange, 2004). Our data also demonstrated that RAP1 negatively regulated TERRA expression, which is in accordance with a study in lower eukaryotes rather than human immortalized cell lines (Kabir et al., 2014; Nanavaty et al., 2017). Thus, our data not only highlight the importance of using human stem cell models to study the role of RAP1 in telomere regulation, but also support a conserved function for RAP1 from yeast to human.

In addition to its telomere-dependent function, mammalian RAP1 plays a role in regulating gene expression. However, most past studies have failed to unravel the underlying mechanisms (Yang et al., 2011; Kabir et al., 2014; Martinez et al., 2016) with the exception of one study, in which RAP1 protected mice from obesity by promoting the transcription of Ppara and Pgc1a by directly binding to the upstream regulatory regions of these genes (Martinez et al., 2010, 2013). In our study, RAP1 bound to the promoter of $R E L N$ and kept it from being hypermethylated in hMSCs and hNSCs, which facilitated the transcription of RELN and accounted at least in part for the delayed senescence in hMSCs. These results provide a novel explanation for how RAP1 regulates the expression of its target genes at the epigenetic level. While how RAP1 regulates DNA methylation is unclear, which warrants further investigation, it is possible that RAP1 per se functions as a demethylase, promotes the activity of certain DNA demethylases, or prevents certain DNA methylases from acting on RELN promoter; it is also possible that RAP1 affects the function of DNA methylases or demethylases by changing chromatin conformation nearby.

Emerging evidences support the notion that stem cell attrition is closely associated with aging and various age-related diseases (Lopez-Otin et al., 2013; Liu et al., 2014; Goodell and Rando, 2015; Zhang et al., 2015; Kubben et al., 2016; Pan et al., 2016; Yang et al., 2017; Wang et al., 2018b; Wu et al., 2018). Here, we highlight an important role for RAP1 in regulating hMSC aging, potentially through both telomere- 


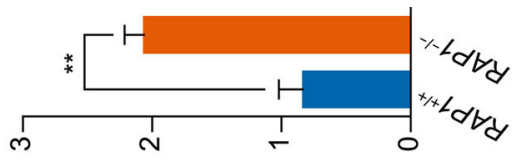

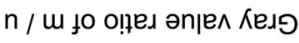

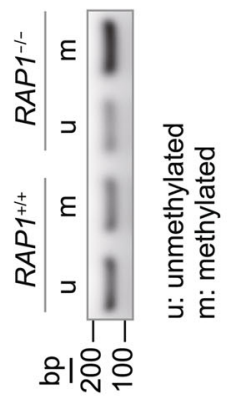

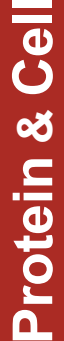

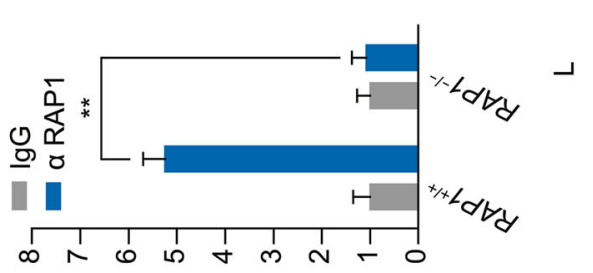

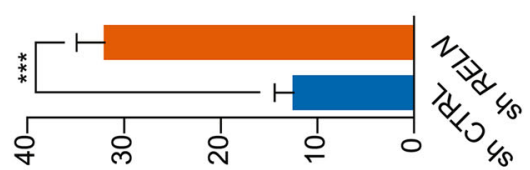

(\%) s||әว ә^!!!sod- $\angle 9 ! Y$

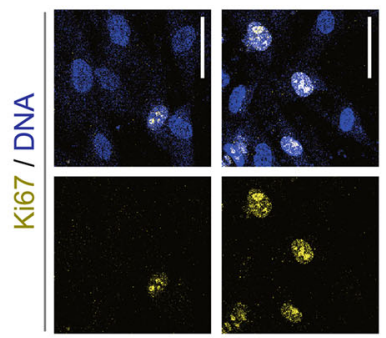

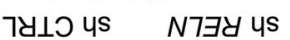

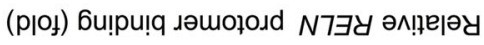

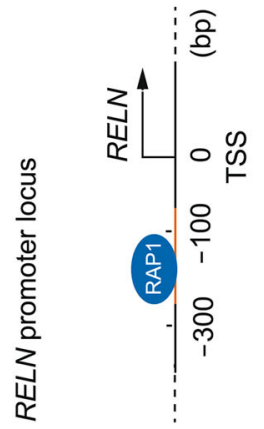

I

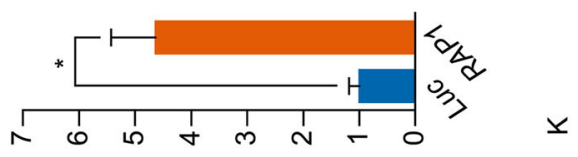

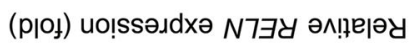

0

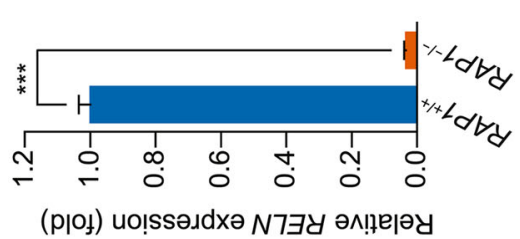

レ

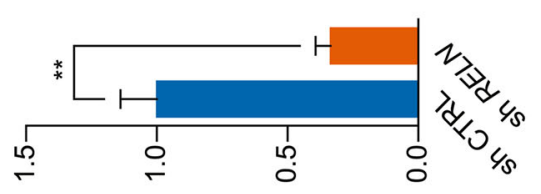

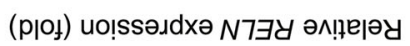

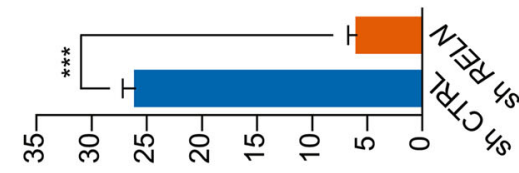

(\%) s॥əo ә^!!! sod ןe6- $\delta-\forall S$

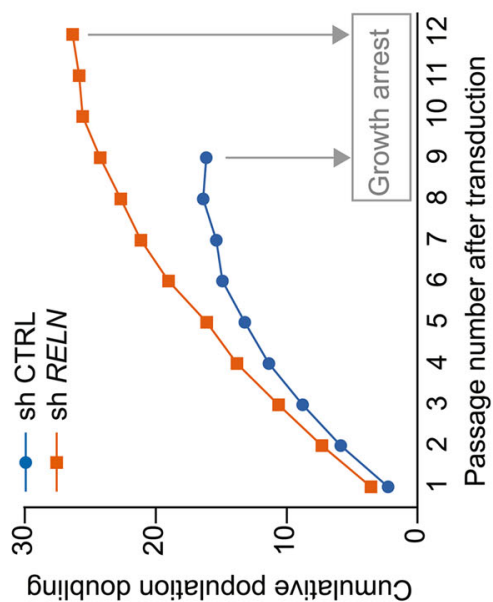

бu!|qnop uo!̣e|ndod әм!̣e|numo

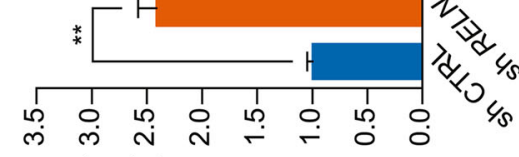

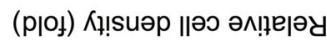

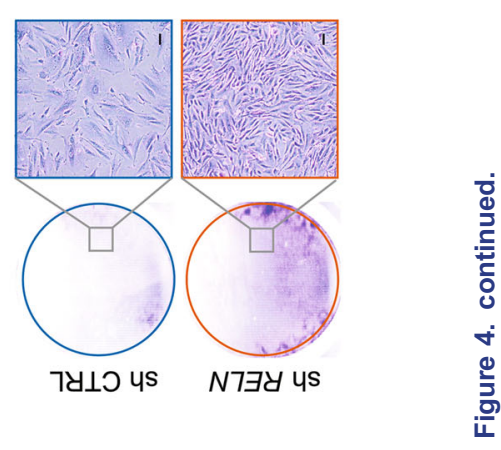


A

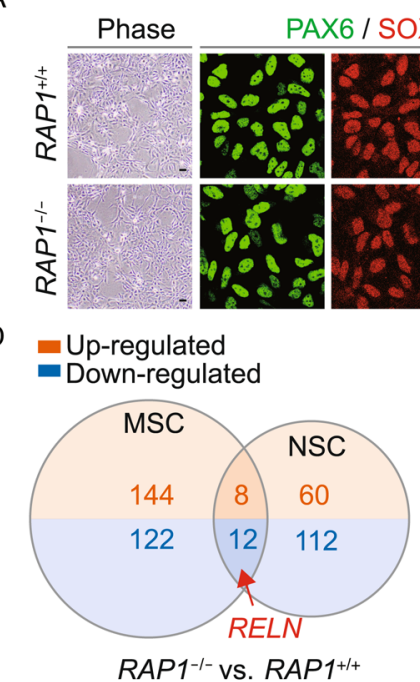

F
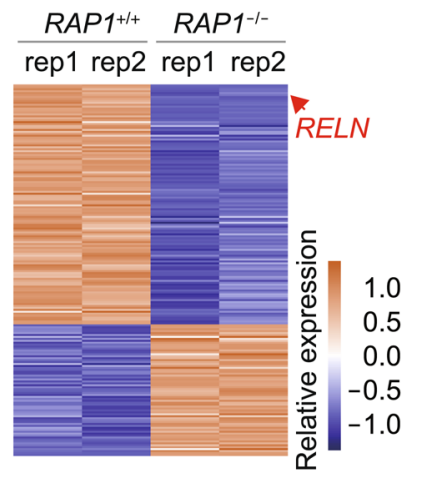

J
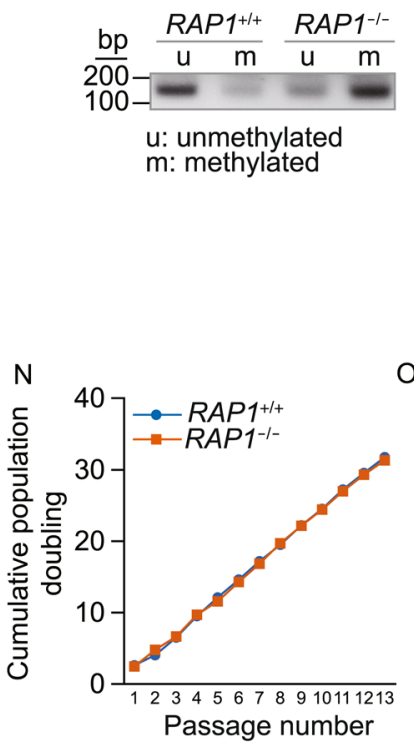

0
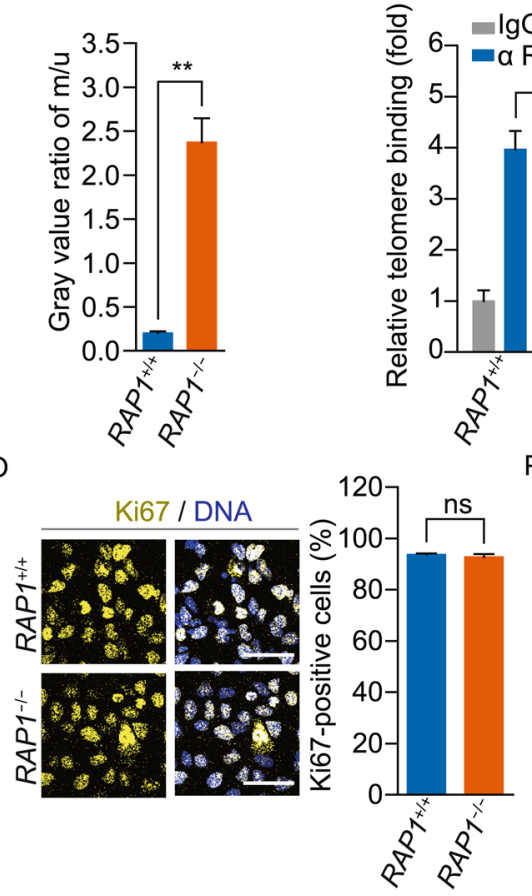

E
B

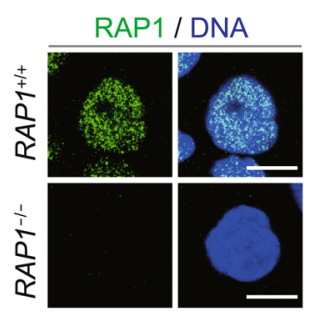

C

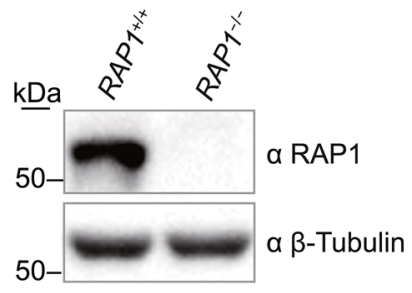

Top 5 enriched biological process GO terms (RAP1-/- vs. $\left.R A P 1^{+/+}\right)$

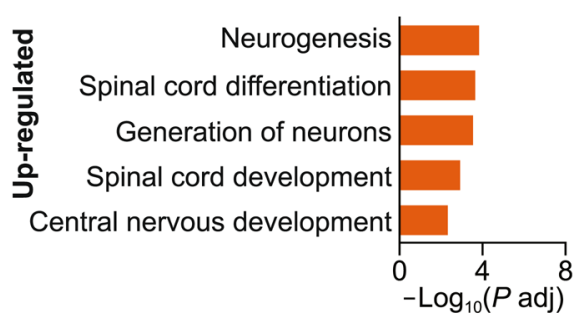

G

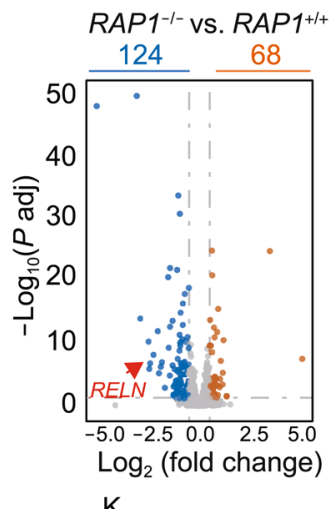

$\mathrm{K}$
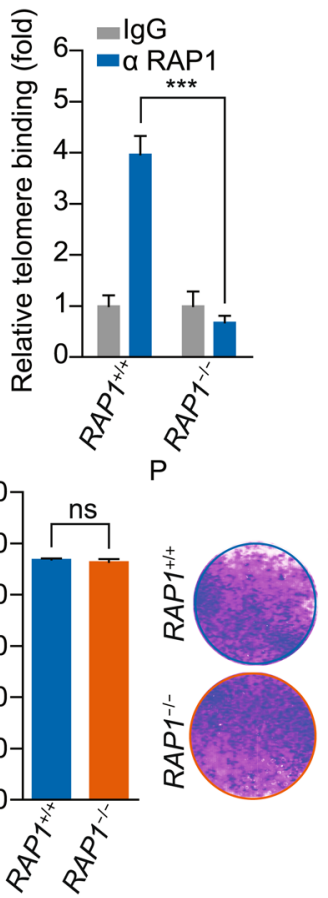

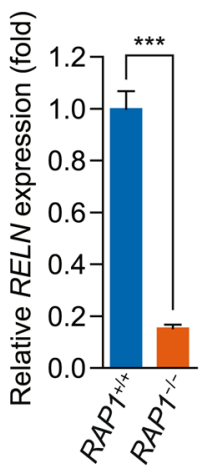

Southern blotting

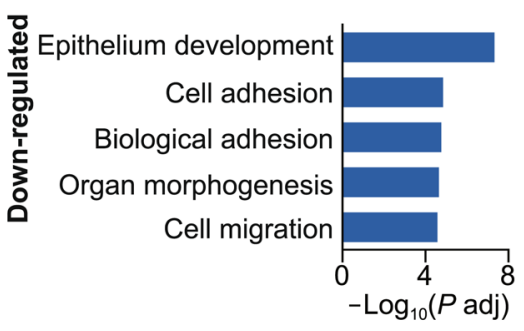

I

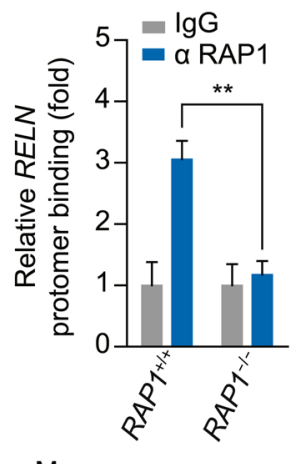

$M$

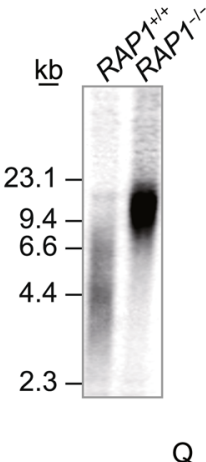

qPCR
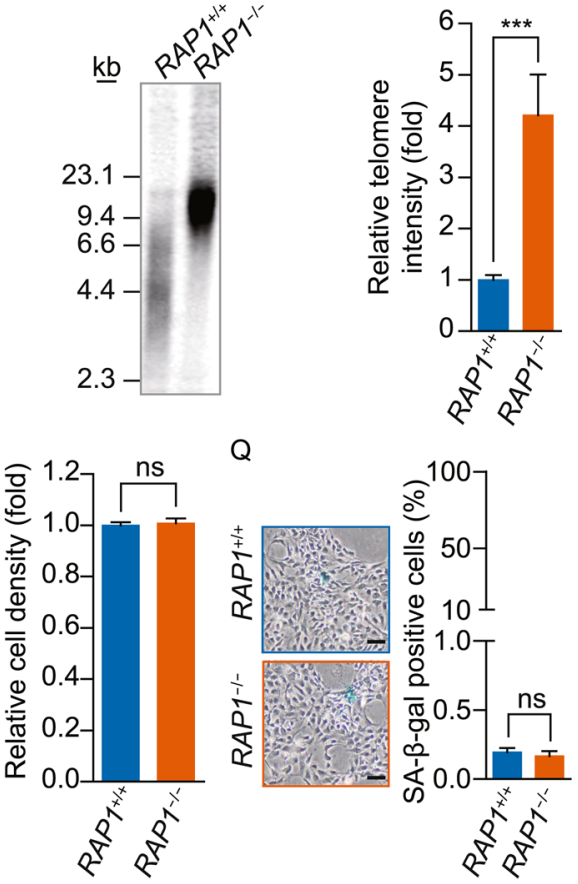
Figure 5. RAP1 deficiency did not influence the proliferation or senescence of hNSCs. (A) Brightfield and immunofluorescence micrographs of WT and $R A P 1^{-1-}$ hNSCs showed normal morphology and expression of the NSC markers PAX6, SOX2 and Nestin. Scale bar, $50 \mu \mathrm{m}$. (B) Immunofluorescence micrographs of RAP1 in WT and RAP1 ${ }^{-1-}$ hNSCs. Scale bar, $10 \mu \mathrm{m}$. (C) Western blotting analysis demonstrated the absence of RAP1 in RAP1 ${ }^{-1-}$ hNSCs. $\beta$-Tubulin was used as a loading control. (D) Venn diagram showing differentially expressed genes in $R A P 1^{-1-}$ vs. WT hMSCs and hNSCs. RELN was labeled by an arrow. (E) Biological process GO enrichment analysis of differentially expressed genes in $R A P 1^{-1-}$ vs. WT hNSCs. The top 5 enriched biological process $\mathrm{GO}$ terms were shown. $(\mathrm{F})$ Heatmap of differentially expressed genes in $R A P 1^{-1-}$ vs. WT hNSCs. RELN was labeled by an arrow. (G) Volcano plot of differentially expressed genes in $R A P 1^{-1-}$ vs. WT hNSCs. $R E L N$ was labeled by an arrow. (H) qRT-PCR analysis verified that $R E L N$ was downregulated in $R A P 1^{-1-} \mathrm{hNSCs}$. Data were presented as the mean \pm SEM, $n=3 .{ }^{* *} P<0.001$. (I) ChIP-PCR of RAP1 enrichment at the RELN promoter region in WT and $R A P 1^{-1-}$ hNSCs. Data were presented as the mean \pm SEM, $n=3$. ${ }^{* *} P<0.01$. (J) Methylation-specific PCR analysis of the RELN promoter in WT and $R A P 1^{-1-}$ hNSCs demonstrated hypermethylation of the RELN promoter in $R A P 1^{-1-}$ hNSCs. Data were presented as the mean \pm SEM, $n=3$. ${ }^{* *} P<0.01$. (K) ChIP-PCR analysis of RAP1 enrichment at the telomeres in $\mathrm{WT}$ and $R A P 1^{-1-} \mathrm{hNSCs}$. Data were presented as the mean \pm SEM, $n=3 .{ }^{* \star *} P<0.001$. (L) Terminal restriction fragment analysis of WT and $R A P 1^{-1-}$ hNSCs by Southern blotting demonstrated the elongated telomere length in $R A P 1^{-1-}$ hNSCs. (M) Telomere length analysis of WT and $R A P 1^{-1-}$ hNSCs by qPCR. Data were presented as the mean $\pm \mathrm{SEM}, n=3$. ${ }^{* * *} P<0.001$. (N) Cell growth curves of WT and $R A P 1^{-1-}$ hNSCs showed comparable proliferation ability of WT and $R A P 1^{-1-}$ hNSCs. (O) Immunostaining of the proliferation marker Ki67 in WT and $R A P 1^{-1-}$ hNSCs. Scale bar, $50 \mu \mathrm{m}$. Data were presented as the mean \pm SEM, $n=6$. NS, not significant. (P) Clonal expansion analysis of WT and $R A P 1^{-1-}$ hNSCs. Data were presented as the mean \pm SEM, $n=3$. NS, not significant. (Q) SA- $\beta$-gal staining of WT and $R A P 1^{-1-}$ hNSCs. Scale bar, $50 \mu \mathrm{m}$. Data were presented as the mean \pm SEM, $n=6$. NS, not significant.

dependent and telomere-independent functions. These new findings may open new avenues of research to better understand the mechanisms of human stem cell attrition and identify new strategies to treat aging-associated disorders.

\section{MATERIALS AND METHODS}

\section{Cell culture}

Human H9 (WT) and RAP1 ${ }^{-1-}$ hESCs were cultured on mouse embryonic fibroblasts (MEFs) inactivated by mitomycin C (Selleck) in hESC medium (DMEM/F12 (Invitrogen) supplemented with $20 \%$ Knockout Serum Replacement (Invitrogen), $2 \mathrm{mmol} / \mathrm{L}$ GlutaMAX (Invitrogen), $0.1 \mathrm{mmol} / \mathrm{L}$ non-essential amino acids (NEAA,
Invitrogen), 1\% penicillin/streptomycin (Invitrogen), $55 \mu \mathrm{mol} / \mathrm{L} \beta$ mercaptoethanol (Invitrogen) and $10 \mathrm{ng} / \mathrm{mL}$ FGF-2 (Joint Protein Central)) (Fu et al., 2016) or on Matrigel (BD Biosciences) in mTeSR medium (STEMCELL Technology). hMSCs were cultured on $0.1 \%$ gelatin (Sigma) in hMSC culture medium (MEMa (Invitrogen) supplemented with $10 \%$ fetal bovine serum (FBS, Gemcell, Cat. No. 100-500, lot. No. A77E01F), $2 \mathrm{mmol} / \mathrm{L}$ GlutaMAX, $0.1 \mathrm{mmol} / \mathrm{L}$ NEAA, $1 \%$ penicillin/streptomycin and $1 \mathrm{ng} / \mathrm{mL}$ FGF-2). hNSCs were cultured in plates coated by Matrigel in neural stem cell maintenance medium (50\% Advanced DMEM/F12 (Invitrogen) and 50\% Neurobasal (Invitrogen), supplemented with 1\% N2 (Invitrogen), 2\% B27 (Invitrogen), $2 \mathrm{mmol} / \mathrm{L}$ GlutaMAX, $0.1 \mathrm{mmol} / \mathrm{L}$ NEAA, 1\% penicillin/ streptomycin, $10 \mathrm{ng} / \mathrm{mL}$ human leukemia inhibitory factor (Millipore), $3 \mu \mathrm{mol} / \mathrm{L} \mathrm{CHIR99021} \mathrm{(Selleck)} \mathrm{and} 2 \mu \mathrm{mol} / \mathrm{L}$ SB431542 (Selleck)). Additionally, $10 \mu \mathrm{mol} / \mathrm{L}$ ROCK inhibitor Y-27632 (Sigma) was added prior to re-plating for each passage before passage 6. HEK293T cells were cultured in 293T culture medium (high glucose DMEM (HyClone) supplemented with 10\% FBS (Gemini), 2 mmol/L GlutaMAX, $0.1 \mathrm{mmol} / \mathrm{L} \mathrm{NEAA}$ and $1 \%$ penicillin/streptomycin). All cells were cultured in an incubator at $37^{\circ} \mathrm{C}$ with $5 \% \mathrm{CO}_{2}$.

Generation of RAP1-knockout $\left(\mathrm{RAP1}^{-l^{-}}\right)$hESCs via the CRISPR/ Cas9 technique

Gene editing via the CRISPR/Cas9 technique was slightly modified from a previously published protocol (Wang et al., 2018b). The RAP1 guide RNA (gRNA) 5'-TGGGTGAATGAGCACGTCCT-3' was cloned into the gRNA-Cloning Vector (Addgene, \#41824). The donor vector contained homology arms and a neo cassette flanked with two FRT sites for HR (Pan et al., 2016). $5 \times 10^{6}$ H9 ESCs pretreated with $10 \mu \mathrm{mol} / \mathrm{L}$ Y-27632 were mixed with three plasmids (sgRNA, donor and Cas9 expression vectors) and then electroporated. Post-electroporated cells were plated on G418-resistant MEF feeders with $10 \mu \mathrm{mol} / \mathrm{L}$ Y-27632. Once hESC clones formed, cell screening was performed by the addition of $100 \mu \mathrm{g} / \mathrm{mL}$ G418 (Sigma). The neo cassette was removed as previously described (Duan et al., 2015). The clones were picked for expansion and verified by genomic PCR. The primers for the HR donor vector construction were 5'-CTATAGGGCGAATTGGGCCC AGCCTCT ATTACCGTCTCTTGTCTGTTGCAT-3' (forward) and 5'-CT GGCGGCCGCTCGAGGGCCACGTACCACAATCCACCAATATAC CAT-3' (reverse) for the left arm and 5'-TTACTAGTGGATCCGAGCTCTGGAAAATGGGACTGATCTGGGCTTCAGAC-3' (forward) and 5'-ATTACGCCAAGCTTGGTACCTCACCACATCTCCAA TACCCACCAATGCCTA-3' (reverse) for the right arm. The primers for clone identification were P1: 5'-TTGGCAAAAGTCAATACAATGGGTAATATCCAAAG-3' (forward), P2: 5'-GGGCATTTTGACAATATCTGATGACATTTATAACG-3' (forward). P3: 5'-AAAACTCC CTCTTGCTGCCCCTTTGT-3' (forward), P4: 5'-GTGGATTGTGGTACGTGGCCCAGATCTGCC-3' (forward), P5: 5'-TAACATACCACAACСTCСTCAAACTCCCGG-3' (reverse), P6: 5'-TGTCCTGCCA AAAACTAAAAGCTTTGTGA-3' (reverse), and P7: 5'-TTTGACTTCACTCTCAAGACTGTAAGCTCCT -3' (reverse).

\section{Generation and characterization of hMSCs}

hMSCs were derived from hESCs as described previously (Pan et al., 2016). In brief, embryoid bodies (EBs) first formed from hESC 

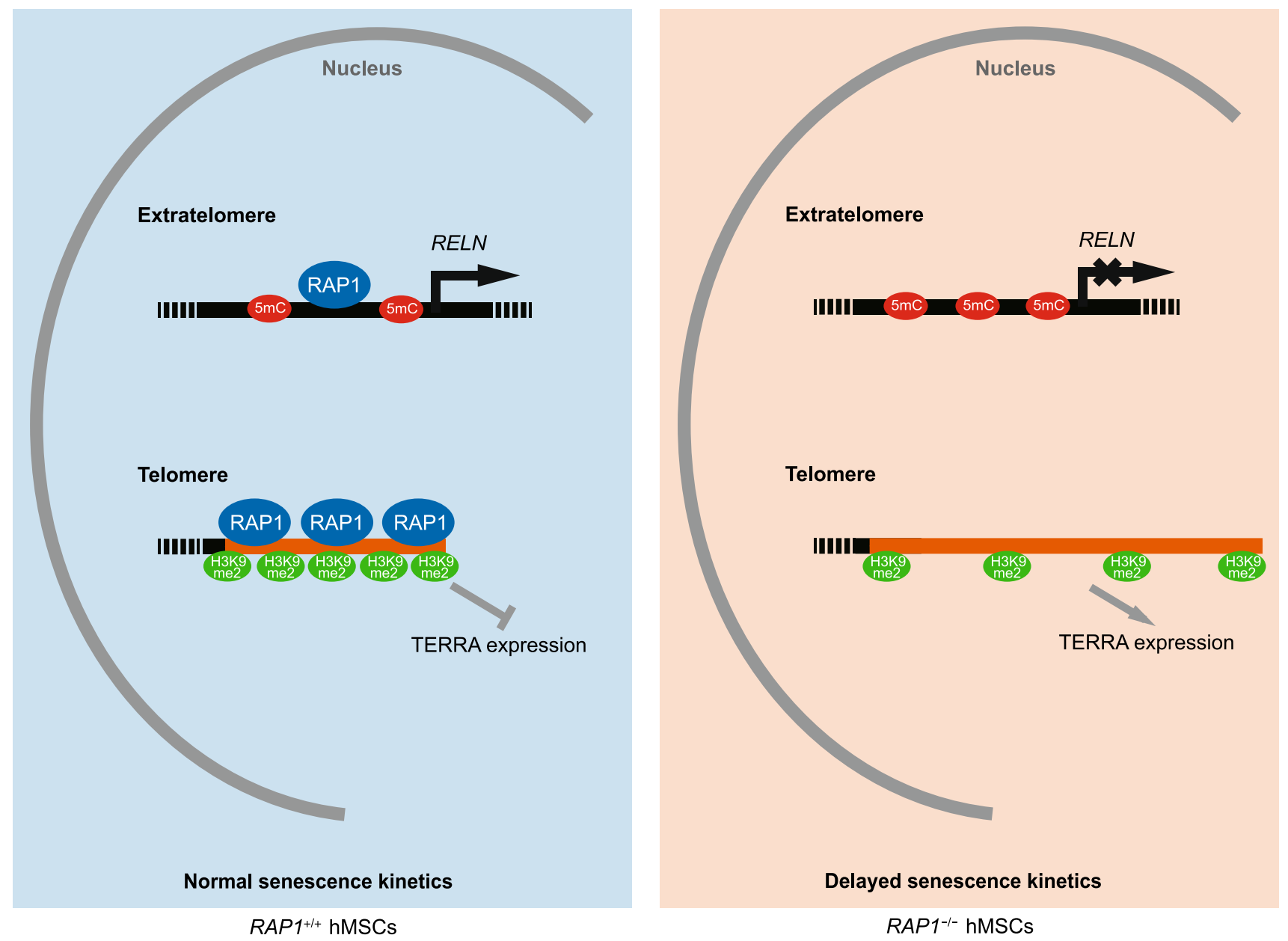

Figure 6. A proposed model showing how RAP1 regulates hMSC homeostasis.

clones in an ultralow attachment 6-well plate (Corning) in low FGF-2 hESC medium and then were transferred to a plate coated by Matrigel in hMSC differentiation medium (hMSC culture medium supplemented with additional $9 \mathrm{ng} / \mathrm{mL}$ FGF-2 and $5 \mathrm{ng} / \mathrm{mL}$ TGF- $\beta$ (HumanZyme)). After 7 to 10 days, the cells became confluent and were reseeded into dishes coated by gelatin in hMSC culture medium. CD73, CD90 and CD105 tri-positive cells were sorted as hMSCs with the aid of flow cytometry. The following antibodies were used: anti-CD73-PE (BD Biosciences, 550257), anti-CD90-FITC (BD Biosciences, 555595) and anti-CD105-APC (eBioscience, 17-1057-42). The differentiation abilities of hMSCs were tested by futher differentiation into chondrocytes, adipocytes and osteoblasts (Liu et al., 2014) detected by toluidine blue (chondrocytes), oil red O (adipocytes) and von Kossa (osteoblasts) staining, respectively.

\section{Generation and characterization of hNSCs}

hNSCs were derived from hESCs as described previously (Duan et al., 2015). Briefly, hESCs were cultured on MEF feeders in neural induction medium-1 (neural stem cell maintenance medium supplemented with $1 \mu \mathrm{mol} / \mathrm{L} \mathrm{CHIR99021,} 1 \mu \mathrm{mol} / \mathrm{L}$ SB431542, $2 \mu \mathrm{mol} / \mathrm{L}$ dorsomorphin (Sigma) and $0.1 \mu \mathrm{mol} / \mathrm{L}$ Compound $\mathrm{E}$ (EMD Chemicals Inc.)) for two days and then neural induction medium-2 (neural induction medium-1 without dorsomorphin) for five days. The cells were subsequently cultured in plates coated by Matrigel in neural stem cell maintenance medium. The hNSC markers PAX6, SOX2 and Nestin were detected by immunofluorescence microscopy. The differentiation ability of hNSCs towards neurons was evaluated by MAP2 and TUJ1 immunostaining (Zhang et al., 2018).

\section{Generation of organoid-like cerebral structures}

Organoid-like cerebral structures were differentiated from hESCs as described previously (Lancaster et al., 2013; Lancaster and Knoblich, 2014). Briefly, hESCs cultured on MEF feeders were digested into single cells. EBs were formed from $2 \times 10^{4}$ cells in a U-bottom ultralow attachment 96-well plate (Corning) in low FGF-2 hESC medium supplemented with $50 \mu \mathrm{mol} / \mathrm{L} \mathrm{Y}-27632$. After four days, FGF-2 and Y-27632 were deprived for another two days. Then, the EBs were transferred to ultralow attachment 24-well plates (Corning) in neural induction medium (DMEM/F12 supplemented with $1 \% \mathrm{~N} 2$, $2 \mathrm{mmol} / \mathrm{L}$ GlutaMAX, $0.1 \mathrm{mmol} / \mathrm{L}$ NEAA, $1 \%$ penicillin/streptomycin and $1 \mu \mathrm{g} / \mathrm{mL}$ heparin (Selleck)) for four days. The EBs were packaged into Matrigel droplets and further cultured in 6-well plates in cerebral organoid differentiation medium (50\% DMEM/F12 and $50 \%$ Neurobasal, with additional $0.5 \% \mathrm{~N} 2,1 \%$ B27 without vitamin A 
(Invitrogen), $2 \mathrm{mmol} / \mathrm{L}$ GlutaMAX, $0.05 \mathrm{mmol} / \mathrm{L}$ NEAA, 1\% penicillin/ streptomycin, $2.75 \mu \mathrm{g} / \mathrm{mL}$ insulin (Sigma) and $50 \mu \mathrm{mol} / \mathrm{L} \beta$-mercaptoethanol (Sigma)) for four days. Then, the plates were placed on an orbital shaker (shaking at $85 \mathrm{rpm}$ ) installed in the incubator, and B27 without vitamin A was replaced by normal B27. After approximately 40 days, the structures were fixed by $4 \%(w / v)$ paraformaldehyde for cryosectioning and immunostaining or collected directly for RNA extraction.

\section{Lentivirus production}

Lentiviruses were expressed and purified as described previously (Duan et al., 2015). To construct the RAP1-overexpression lentiviral vector, the cDNA of flag-RAP1 was amplified by PCR with the primers 5'-CCGCTCGAG ATGGACTACAAGGACGACGACGACAAG GGCGCGGAGGCGATGGATTTGGG-3' (forward) and 5'-CGACG CGTTTATTTCTTTCGAAATTCAATCCTCCGAGC-3' (reverse), cleaved by Xhol (NEB) and Mlul (NEB), and cloned into the pLE4 vector (a kind gift from Doctor Tomoaki Hishida) pre-cleaved by Xhol and Mlul. To generate the lentiviral vector encoding shRNA targeting $R E L N$, the annealed fragment from the oligos 5'-CGCGT GCACGG ATGAAAGGAGTCTTATTCAAGAGATAAGACTCCTTTCATCCGTG CTTTTTTGGAAAT-3' (forward) and 5'-CGATTTCCAAAAAA GCAC GGATGAAAGGAGTCTTATCTCTTGAATAAGACTCCTTTCATCCG TGCA-3' (reverse) was phosphorylated by T4 Polynucleotide Kinase (NEB) and cloned into the pLVTHM vector pre-cleaved by Clal (NEB) and Mlul. For lentiviral packaging, HEK293T cells were cultured up to $95 \%$ confluency and then co-transfected with the overexpression or shRNA vectors, along with psPAX2 (Addgene, $\# 12260$ ) and pMD2.G (Addgene, \#12259). Two days later, the culture medium was collected and concentrated by ultracentrifugation at $4{ }^{\circ} \mathrm{C}$. The lentiviral particles were used for transduction with $4 \mu \mathrm{g} / \mathrm{mL}$ polybrene.

\section{Clonal expansion assay}

Two thousand cells were seeded in a well of a 12-well plate (Corning) and cultured for approximately 10 days. Only for hESCs, $10 \mu \mathrm{mol} / \mathrm{L} \mathrm{Y}-27632$ was added on the first day of cell passaging and removed after $24 \mathrm{~h}$. The relative colony number and relative cell integral density was calculated by ImageJ software after crystal violet staining.

\section{SA- $\beta$-gal staining}

SA- $\beta$-gal staining was performed as described previously (DebacqChainiaux et al., 2009; Wu et al., 2018). In brief, cells were fixed with fixation buffer containing $2 \%(w / v)$ formaldehyde and $0.2 \%(w / v)$ glutaraldehyde for $5 \mathrm{~min}$. Then, the cells were treated with staining buffer containing $1 \mathrm{mg} / \mathrm{mL}$ X-gal overnight at $37{ }^{\circ} \mathrm{C}$. Stained cells were observed by optical microscope and the percentage of positive cells was analyzed by ImageJ software.

\section{Cell cycle analysis}

Cell cycle analysis was performed as described previously (Wang et al., 2018b). In brief, cells were fixed by $70 \%$ precooled ethanol at least overnight and then treated with staining buffer containing $0.1 \%$ Triton X-100, $0.2 \mathrm{mg} / \mathrm{mL}$ RNase A and $0.02 \mathrm{mg} / \mathrm{mL}$ propidium iodide at $37^{\circ} \mathrm{C}$ for $30 \mathrm{~min}$. Then, the cells were analyzed by an LSRFortessa cell analyzer (BD), and data were measured by ModFit software.

\section{Cell viability analysis}

MTS analysis of hMSCs was performed as described previously (Pan et al., 2016). In brief, cells at 90\% confluence in 96-well plates (Corning) were treated with different stressors for $24 \mathrm{~h}$. Cell vitality was measured by MTS colorimetry.

\section{In vitro cell migration analysis}

Transwell assay of hNSCs was performed as described previously (Duan et al., 2015). In brief, $2.5 \times 10^{4}$ cells were resuspended by $100 \mu \mathrm{L}$ basal medium (50\% Advanced DMEM/F12 and 50\% Neurobasal) and seeded on the top of transwell (Corning) with $500 \mu \mathrm{L}$ neural stem cell maintenance medium added to the bottom. $24 \mathrm{~h}$ later, the cells at the lower surface of transwells were calculated by ImageJ software after crystal violet staining.

\section{Genomic and bisulfite PCR}

Genomic DNA was extracted by a DNA extraction kit (TIANGEN). General PCR was performed with the PrimeSTAR HS DNA Polymerase with GC Buffer Kit (TAKARA) and a 96-well thermal cycler (Applied Biosystems). Bisulfite conversion of genomic DNA was carried out with the EZ DNA Methylation-Lightning Kit (ZYMO Research). Bisulfite PCR was carried out with LA Taq Hot Start Version (TAKARA). Detection of the methylation level of the OCT4 promoter was performed as described previously (Yu et al., 2007; Duan et al., 2015). In brief, the PCR products were recycled by the QIAquick Gel Extraction Kit (Qiagen), cloned into the T-vector (Takara) and sequenced with the universal primer M13. The methylation level of the RELN promoter was detected by methylation-specific PCR (Omura et al., 2008; Vincent et al., 2011) with proper primers (Sato et al., 2006). Image $\mathrm{J}$ software was used to calculate the gray value ratio of methylated/unmethylated bands.

\section{RT-PCR}

For general RT-PCR, total RNA was extracted by TRIzol (Invitrogen). For RT-PCR of TERRA, total RNA was extracted by the RaPure Total RNA Micro Kit (Magen) with DNA removal on the column. Then, cDNA was synthesized with the GoScript Reverse Transcription System (Promega). The semi-quantitative RT-PCR method was the same as that for general PCR, while qRT-PCR was performed using THUNDERBIRD qPCR Mix (TOYOBO) and the CFX384 Real-Time System (BioRad). The primers for TERRA detection were described previously (Feretzaki and Lingner, 2017). Primers to detect the exon 2 of RAP1 were P8: 5'-GGGCCAGGAGCATAAGTACC-3' (forward) and P9: 5'-GGAGTTCTCTTATTCTGTGGTTCC-3' (reverse) (Hohensinner et al., 2016); primers for the internal reference for general qRT-PCR, 18S rRNA, were 5'-GTAACCCGTTGAACCCCATT-3' (forward) and 5'-CCATCCAATCGGTAGTAGCG-3' (reverse) (Ren et al., 2017a). Other primer sequences were obtained from published articles (Takahashi et al., 2007; Yang et al., 2011; Zhang et al., 2015) or selected from PrimerBank (https://pga.mgh.harvard.edu/primerbank/). 


\section{Western blotting}

Cells were lysed in $2 \times$ SDS-sample buffer without glycerol and $\beta$ mercaptoethanol and heated at $95{ }^{\circ} \mathrm{C}$ for $10 \mathrm{~min}$ (Li et al., 2001). Then, the samples were quantified via a BCA (bicinchoninic acid) protein quantification assay. Generally, lysate containing $30 \mu \mathrm{g}$ of total protein was loaded onto an SDS-PAGE gel for electrophoresis and then electrotransferred to a PVDF membrane (Millipore). After blocking with $5 \%(w / v)$ nonfat powdered milk (BBI Life Sciences), the membrane was successively incubated with primary and HRPconjugated secondary antibodies and blotted by SuperSignal West Femto Maximum Sensitivity Substrate (Thermo Fisher). Finally, imaging and quantification were performed with the ChemiDoc XRS system (Bio-Rad) and Image Lab software. The primary antibodies used for Western blotting in this study were anti-RAP1 (Santa Cruz, sc53434), anti-P16 (BD, 550834), anti-P21 (CST, 2947s), anti- $\beta$ actin (Santa Cruz, sc69879) and anti- $\beta$-tubulin (Santa Cruz, sc5274). To confirm that RAP1 was completely knocked out in hESCs, we also used another RAP1 antibody (\#765) that detects potential truncations of RAP1 (Kabir et al., 2014), which was a gift from $\mathrm{T}$. de Lange.

\section{Telomere length analysis}

Measurement of telomere length by $\mathrm{qPCR}$ and Southern blotting was conducted as described previously (Cawthon, 2002; Lai et al., 2016). However, capillary transfer was used. Measurement of telomere length by flow FISH was performed by imitating a 3D-FISH/ immunolabeling protocol (Ren et al., 2017a). Briefly, adherent hMSCs were digested and neutralized. The cells were then incubated with $4 \%(w / v)$ paraformaldehyde, $0.4 \%(v / v)$ Triton X-100 in PBS, $100 \mu \mathrm{g} / \mathrm{mL}$ RNase A in PBS, and 20\% ( $v / v)$ glycerol in PBS and then heated to denature genomic DNA and hybridized with Cy3labeled telomere PNA probe (Panagene) overnight at $37{ }^{\circ} \mathrm{C}$ in a hybridization oven (UVP). The samples were measured with an LSRFortessa cell analyzer (BD), and data were analyzed by FlowJo software.

\section{Immunofluorescence microscopy}

Cells were successively treated for $30 \mathrm{~min}$ with $4 \%(w / v)$ paraformaldehyde, $0.4 \%(v / v)$ Triton X-100 in PBS and dilute donkey serum (Jackson ImmunoResearch Labs), and then incubated with primary antibodies overnight at $4{ }^{\circ} \mathrm{C}$ and corresponding fluorescent secondary antibodies as well as Hoechst 33342 (Invitrogen) at room temperature for $1 \mathrm{~h}$. The primary antibodies used were anti-RAP1 (Santa Cruz, sc53434), anti-OCT3/4 (Santa Cruz, sc5279), antiSOX2 (Santa Cruz, sc17320), anti-NANOG (Abcam, ab21624), antiTUJ1 (Sigma, T2220), anti-SMA (Sigma, A5228), anti-FOXA2 (CST, 8186), anti-Ki67 (Vector, VP-RM04), anti-PAX6 (Covance, PRB278P), anti-Nestin (BD, 560422), anti-MAP2 (Sigma, M4403) and anti-Reelin (MBL, D223-3).

\section{ChIP}

The ChIP assay was slightly modified from versions described previously (Dahl and Collas, 2008; Zhang et al., 2018). In brief, cells were harvested and crosslinked by $1 \%(v / v)$ formaldehyde for $15 \mathrm{~min}$ (RAP1) or $8 \mathrm{~min}$ (H3K9me2), and then termination of crosslinking was performed by $125 \mathrm{mmol} / \mathrm{L}$ glycine for $5 \mathrm{~min}$. After washing with PBS, the cells were lysed and sonicated to generate DNA fragments. The product was incubated with antibody binding beads overnight at $4{ }^{\circ} \mathrm{C}$. Then, the supernatant was removed, and the beads were decrosslinked for $2 \mathrm{~h}$ at $68^{\circ} \mathrm{C}$. The DNA was recycled and quantified by a qPCR assay. Antibodies for ChIP included antiRAP1 (Santa Cruz, sc53434), anti-H3K9me2 (Abcam, ab1220) and mouse IgG (Santa Cruz, sc2025) as a negative control. The primers for the RELN promoter of RAP1 binding locus were $5^{\prime}$-CGAGCCAGCCCGAGA-3' (forward) and 5'-GTCGTCTGCCGCCTCC-3' (reverse).

\section{Animal experiments}

Teratoma assay was carried out as described previously (Lensch et al., 2007). Briefly, approximately $5 \times 10^{6} \mathrm{hESCs}$ on feeder layers were injected into the groin cavities of NOD/SCID mice (male, 6-8 weeks). After approximately 2 months, the teratomas were taken out and analyzed by immunofluorescence staining. hMSC transplantation assay was carried out as described previously (Yang et al., 2017). In brief, cells were previously transduced with lentiviruses that express luciferase in human cells. A total of $1 \times 10^{6}$ cells were injected into the midportion of the TA muscle of nude mice (male, 6 8 weeks). Every two days after transplantation, mice were treated with D-luciferin and then imaged by an IVIS spectrum imaging system (XENOGEN, Caliper) in AUTO mode. Animal experiments were performed with the approval of the Institute of Biophysics, Chinese Academy of Science (IBP, CAS).

\section{CNV identification}

Genomic DNA of early-passage (P2) hMSCs was extracted by the DNeasy Blood and Tissue Kit (Qiagen).Then, the DNA was fragmented by a Covaris S220 ultrasonicator, and libraries were constructed by using the NEBNext Ultra ${ }^{\text {TM }}$ DNA Library Prep Kit for Illumina (NEB). The samples were clustered by the Truseq PE Cluster Kit V4 and sequenced on an Illumina Hiseq X-ten platform. For CNV analysis, paired end reads were trimmed and aligned to the UCSC hg19 human reference genome by bowtie2 software (v2.2.9) (Langmead and Salzberg, 2012). CNVs were calculated by readCounter and normalized by HMMcopy (v1.20.0) in a 500-kb window (Ha et al., 2012).

\section{RNA-seq library preparation and sequencing}

Total RNA of early-passage hMSCs (P2) or hNSCs (P3) was extracted by TRIzol. Library construction, sequencing and processing of RNA-seq data were carried out as previously described (Geng et al., 2018; Wang et al., 2018a). Briefly, RNA integrity was first qualified by using the RNA Nano 6000 Assay Kit for the Bioanalyzer 2100 system (Agilent Technologies), and then libraries were constructed by using the NEBNext Ultra ${ }^{\mathrm{TM}}$ RNA Library Prep Kit for Illumina (NEB). The samples were clustered on a cBot Cluster Generation System by TruSeq PE Cluster Kit v3-cBot-HS (Illumina) and sequenced on an Illumina Hiseq platform. Raw data were trimmed and then mapped to the UCSC hg19 human genome using hisat2 (v2.0.4) (Kim et al., 2015). The transcriptional level of each gene was counted by HTSeq (v0.6.1) (Anders et al., 2015). 
Differentially expressed genes were calculated by the DESeq2 R package with the cutoff Benjamini-Hochberg adjusted $P$ value ( $P$ adj) of less than 0.05 and $\mid \log _{2}$ (fold change)| of more than 0.5 (Love et al., 2014). GO-BP enrichment analysis was conducted by ToppGene (Chen et al., 2009).

\section{Statistical analysis}

Results were presented as the mean \pm SEM. Graph-Pad Prism software was used to perform a two-tailed Student's $t$-test. Statistical significance was presented as ${ }^{*} P$ values $<0.05$, ${ }^{* *} P$ values $<0.01$ and ${ }^{* * *} P$ values $<0.001$

\section{ACKNOWLEDGEMENTS}

We thank Professor Titia de Lange from the Rockefeller University for sharing a human RAP1 antibody \#765 that detects potential truncations of RAP1. We are grateful to Lei Bai, Qun Chu, Ruijun Bai and Shikun Ma for administrative assistance, to Ruotong Ren, Lina Fu, Zeming Wu, Liping Deng, Pengze Yan and Xiaoqian Zhang for their technical assistance, to Sai Yang (IBP, CAS), Na Li (IBP, CAS), Shuo Guo (IBP, CAS), Xinyi Wu (IBP, CAS), Hui Su (IBP, CAS), Mengfei Wang (IBP, CAS), Shengnan Cui (IBP, CAS) and Chang Guo (IBP, CAS) for management of laboratory animals, to Lei Zhou (IBP, CAS) for providing veterinary care, to Junying Jia (IBP, CAS) and Shuang Sun (IBP, CAS) for their help in the flow cytometry experiments, and to Yihui $\mathrm{Xu}$ (Key Laboratory of Infection and Immunity, IBP, CAS) for her help in optical in vivo imaging. We thank Tongxin Niu at HPC-Service Station in Center for Biological Imaging (IBP, CAS) for the support of bioinformatic analysis. This work was supported by the National Key Research and Development Program of China (2018YFA0107001), the Strategic Priority Research Program of the Chinese Academy of Sciences (XDA16010100), the National Key Research and Development Program of China (2018YFC2000100, 2018YFA0107203, 2017YFA0103304, 2017 YFA0102802, 2015CB964800, 2014CB910503), the National Natural Science Foundation of China (81625009, 81330008, 91749202, 91749123, 31671429, 81671377, 81771515, 31601109, 31601158, 81701388, 81422017, 81601233, 81471414, 81870228, 81822018, 81801399, 31801010, 81801370 and 81861168034), Program of Beijing Municipal Science and Technology Commission (Z151100003915072), Key Research Program of the Chinese Academy of Sciences (KJZDEWTZ-L05), Beijing Municipal Commission of Health and Family Planning (PXM2018_026283_ 000002), Advanced Innovation Center for Human Brain Protection (117212) and the State Key Laboratory of Membrane Biology.

\section{ABBREVIATIONS}

CNV, copy number variation; CRISPR/Cas9, clustered regularly interspaced short palindromic repeat/ CRISPR associated gene 9; EBs, embryoid bodies; EP, early passage; FBS, fetal bovine serum; GO-BP, biological process gene ontology; gRNA, guide RNA; hESCs, human embryonic stem cells; hMSCs, human mesenchymal stem cells; hNSCs, human neural stem cells; HR, homologous recombination; LP, late passage; MEF, mouse embryonic fibroblasts; NEAA, non-essential amino acids; NOD/SCID, non-obese diabetic severe combined immunodeficiency; qRT-PCR, quantitative reverse transcription PCR; RAP1, repressor/activator protein 1; RNA-seq,
RNA sequencing; SA- $\beta$-gal, senescence-associated $\beta$-galactosidase; TA, tibialis anterior; TERF2IP, TERF2 interaction protein; TERRA, telomeric repeat-containing RNA; WT, wild type.

Data availability The raw data and processed data reported in this paper were deposited into the GEO database with the accession number GSE122654.

\section{COMPLIANCE WITH ETHICS GUIDELINES}

Xing Zhang, Zunpeng Liu, Xiaoqian Liu, Si Wang, Yiyuan Zhang, Xiaojuan He, Shuhui Sun, Shuai Ma, Ng Shyh-Chang, Feng Liu, Qiang Wang, Xiaoqun Wang, Lin Liu, Weiqi Zhang, Moshi Song, Guang-Hui Liu and Jing Qu declare that they have no conflict of interest. All institutional and national guidelines for the care and use of laboratory animals were followed.

\section{OPEN ACCESS}

This article is distributed under the terms of the Creative Commons Attribution 4.0 International License (http://creativecommons.org/ licenses/by/4.0/), which permits unrestricted use, distribution, and reproduction in any medium, provided you give appropriate credit to the original author(s) and the source, provide a link to the Creative Commons license, and indicate if changes were made.

\section{REFERENCES}

Anders S, Pyl PT, Huber W (2015) HTSeq-a Python framework to work with high-throughput sequencing data. Nat Methods 31:166-169

Arnoult N, Van Beneden A, Decottignies A (2012) Telomere length regulates TERRA levels through increased trimethylation of telomeric H3K9 and HP1alpha. Nat Struct Mol Biol 19:948-956

Bandaria JN, Qin P, Berk V, Chu S, Yildiz A (2016) Shelterin Protects Chromosome Ends by Compacting Telomeric Chromatin. Cell 164:735-746

Blackburn EH (2001) Switching and signaling at the telomere. Cell 106:661-673

Blackburn EH, Epel ES, Lin J (2015) Human telomere biology: A contributory and interactive factor in aging, disease risks, and protection. Science 350:1193-1198

Bourgeron T, Xu Z, Doumic M, Teixeira MT (2015) The asymmetry of telomere replication contributes to replicative senescence heterogeneity. Sci Rep 5:15326

Calado RT, Dumitriu B (2013) Telomere dynamics in mice and humans. Semin Hematol 50:165-174

Cawthon RM (2002) Telomere measurement by quantitative PCR. Nucleic Acids Res 30:e47

Chen J, Bardes EE, Aronow BJ, Jegga AG (2009) ToppGene Suite for gene list enrichment analysis and candidate gene prioritization. Nucleic acids Res 37:W305-311

Dahl JA, Collas P (2008) A rapid micro chromatin immunoprecipitation assay (microChIP). Nat Protoc 3:1032-1045

Debacq-Chainiaux F, Erusalimsky JD, Campisi J, Toussaint $O$ (2009) Protocols to detect senescence-associated beta-galactosidase (SA-betagal) activity, a biomarker of senescent cells in culture and in vivo. Nat Protoc 4:1798-1806 
Di Palma T, Filippone MG, Pierantoni GM, Fusco A, Soddu S, Zannini M (2013) Pax8 has a critical role in epithelial cell survival and proliferation. Cell Death Dis 4:e729

Ding Y, Liang X, Zhang Y, Yi L, Shum HC, Chen Q, Chan BP, Fan H, Liu Z, Tergaonkar $V$ et al (2018) Rap1 deficiency-provoked paracrine dysfunction impairs immunosuppressive potency of mesenchymal stem cells in allograft rejection of heart transplantation. Cell Death Dis 9:386

Doerks T, Copley RR, Schultz J, Ponting CP, Bork P (2002) Systematic identification of novel protein domain families associated with nuclear functions. Genome Res 12:47-56

Donato R, Sorci G, Giambanco I (2017) S100A6 protein: functional roles. Cell Mol Life Sci 74:2749-2760

Duan S, Yuan G, Liu X, Ren R, Li J, Zhang W, Wu J, Xu X, Fu L, Li Y et al (2015) PTEN deficiency reprogrammes human neural stem cells towards a glioblastoma stem cell-like phenotype. Nat Commun 6:10068

Feretzaki M, Lingner J (2017) A practical qPCR approach to detect TERRA, the elusive telomeric repeat-containing RNA. Methods 114:39-45

Ferrara-Romeo I, Martinez P, Blasco MA (2018) Mice lacking RAP1 show early onset and higher rates of DEN-induced hepatocellular carcinomas in female mice. PloS ONE 13:e0204909

Fu L, Xu X, Ren R, Wu J, Zhang W, Yang J, Ren X, Wang S, Zhao Y, Sun $L$ et al (2016) Modeling xeroderma pigmentosum associated neurological pathologies with patients-derived iPSCs. Protein Cell 7:210-221

Geng L, Liu Z, Zhang W, Li W, Wu Z, Wang W, Ren R, Su Y, Wang P, Sun $L$ et al (2018) Chemical screen identifies a geroprotective role of quercetin in premature aging. Protein Cell. https://doi.org/ 10.1007/s13238-018-0567-y

Goodell MA, Rando TA (2015) Stem cells and healthy aging. Science 350:1199-1204

Greider CW (1991) Telomeres. Curr Opin Cell Biol 3:444-451

Ha G, Roth A, Lai D, Bashashati A, Ding J, Goya R, Giuliany R, Rosner J, Oloumi A, Shumansky K et al (2012) Integrative analysis of genome-wide loss of heterozygosity and monoallelic expression at nucleotide resolution reveals disrupted pathways in triple-negative breast cancer. Genome Res 22:1995-2007

Hohensinner PJ, Kaun C, Buchberger E, Ebenbauer B, Demyanets S, Huk I, Eppel W, Maurer G, Huber K, Wojta J (2016) Age intrinsic loss of telomere protection via TRF1 reduction in endothelial cells. Biochim Biophys Acta 1863:360-367

Ishii K, Kubo KI, Nakajima K (2016) Reelin and Neuropsychiatric Disorders. Front Cell Neurosci 10:229

Kabir S, Hockemeyer D, de Lange T (2014) TALEN gene knockouts reveal no requirement for the conserved human shelterin protein Rap1 in telomere protection and length regulation. Cell Rep 9:1273-1280

Kabir S, Sfeir A, de Lange T (2010) Taking apart Rap1: an adaptor protein with telomeric and non-telomeric functions. Cell cycle 9:4061-4067

Kanoh J, Ishikawa F (2001) spRap1 and spRif1, recruited to telomeres by Taz1, are essential for telomere function in fission yeast. Curr Biol 11:1624-1630

Khurana E, Fu Y, Chen J, Gerstein M (2013) Interpretation of genomic variants using a unified biological network approach. PLoS Comput Biol 9:e1002886
Kim D, Langmead B, Salzberg SL (2015) HISAT: a fast spliced aligner with low memory requirements. Nat Methods 12:357-360

Kim H, Li F, He Q, Deng T, Xu J, Jin F, Coarfa C, Putluri N, Liu D, Songyang $Z$ (2017) Systematic analysis of human telomeric dysfunction using inducible telosome/shelterin CRISPR/Cas9 knockout cells. Cell Discov 3:17034

Kubben N, Zhang W, Wang L, Voss TC, Yang J, Qu J, Liu GH, Misteli T (2016) Repression of the antioxidant NRF2 pathway in premature aging. Cell 165:1361-1374

Kundakovic M, Chen Y, Costa E, Grayson DR (2007) DNA methyltransferase inhibitors coordinately induce expression of the human reelin and glutamic acid decarboxylase 67 genes. Mol Pharmacol 71:644-653

Kundakovic M, Chen Y, Guidotti A, Grayson DR (2009) The reelin and GAD67 promoters are activated by epigenetic drugs that facilitate the disruption of local repressor complexes. Mol Pharmacol 75:342-354

Kyrion G, Liu K, Liu C, Lustig AJ (1993) RAP1 and telomere structure regulate telomere position effects in Saccharomyces cerevisiae. Genes Dev 7:1146-1159

Lai TP, Wright WE, Shay JW (2016) Generation of digoxigeninincorporated probes to enhance DNA detection sensitivity. BioTechniques 60:306-309

Lancaster MA, Knoblich JA (2014) Generation of cerebral organoids from human pluripotent stem cells. Nat Protoc 9:2329-2340

Lancaster MA, Renner M, Martin CA, Wenzel D, Bicknell LS, Hurles ME, Homfray T, Penninger JM, Jackson AP, Knoblich JA (2013) Cerebral organoids model human brain development and microcephaly. Nature 501:373-379

Langmead B, Salzberg SL (2012) Fast gapped-read alignment with Bowtie 2. Nat Methods 9:357-359

Lensch MW, Schlaeger TM, Zon LI, Daley GQ (2007) Teratoma formation assays with human embryonic stem cells: a rationale for one type of human-animal chimera. Cell Stem Cell 1:253-258

Li B, de Lange T (2003) Rap1 affects the length and heterogeneity of human telomeres. Mol Biol Cell 14:5060-5068

Li WP, Liu P, Pilcher BK, Anderson RG (2001) Cell-specific targeting of caveolin-1 to caveolae, secretory vesicles, cytoplasm or mitochondria. J Cell Sci 114:1397-1408

Lintas C, Sacco R, Persico AM (2016) Differential methylation at the RELN gene promoter in temporal cortex from autistic and typically developing post-puberal subjects. J Neurodev Disord 8:18

Liu GH, Barkho BZ, Ruiz S, Diep D, Qu J, Yang SL, Panopoulos AD, Suzuki K, Kurian L, Walsh C et al (2011) Recapitulation of premature ageing with iPSCs from Hutchinson-Gilford progeria syndrome. Nature 472:221-225

Liu GH, Suzuki K, Li M, Qu J, Montserrat N, Tarantino C, Gu Y, Yi F, Xu X, Zhang W et al (2014) Modelling Fanconi anemia pathogenesis and therapeutics using integration-free patient-derived iPSCs. Nat Commun 5:4330

Londono-Vallejo JA (2004) Telomere length heterogeneity and chromosome instability. Cancer letters 212:135-144

Lopez-Otin C, Blasco MA, Partridge L, Serrano M, Kroemer G (2013) The hallmarks of aging. Cell 153:1194-1217

Love MI, Huber W, Anders S (2014) Moderated estimation of fold change and dispersion for RNA-seq data with DESeq2. Genome Biol 15:550 
Luo K, Vega-Palas MA, Grunstein M (2002) Rap1-Sir4 binding independent of other Sir, yKu, or histone interactions initiates the assembly of telomeric heterochromatin in yeast. Genes Dev 16:1528-1539

Martinez P, Gomez-Lopez G, Garcia F, Mercken E, Mitchell S, Flores JM, de Cabo R, Blasco MA (2013) RAP1 protects from obesity through its extratelomeric role regulating gene expression. Cell reports 3:2059-2074

Martinez P, Gomez-Lopez G, Pisano DG, Flores JM, Blasco MA (2016) A genetic interaction between RAP1 and telomerase reveals an unanticipated role for RAP1 in telomere maintenance. Aging Cell 15(6):1113-1125

Martinez P, Thanasoula M, Carlos AR, Gomez-Lopez G, Tejera AM, Schoeftner S, Dominguez O, Pisano DG, Tarsounas M, Blasco MA (2010) Mammalian Rap1 controls telomere function and gene expression through binding to telomeric and extratelomeric sites. Nat Cell Biol 12:768-780

Moretti P, Shore D (2001) Multiple interactions in Sir protein recruitment by Rap1p at silencers and telomeres in yeast. Molecular and cellular biology 21:8082-8094

Nabil Fikri RM, Norlelawati AT, Nour El-Huda AR, Hanisah MN, Kartini A, Norsidah K, Nor Zamzila A (2017) Reelin (RELN) DNA methylation in the peripheral blood of schizophrenia. J Psychiatr Res 88:28-37

Nanavaty V, Sandhu R, Jehi SE, Pandya UM, Li B (2017) Trypanosoma brucei RAP1 maintains telomere and subtelomere integrity by suppressing TERRA and telomeric RNA:DNA hybrids. Nucleic Acids Res 45:5785-5796

O'Connor MS, Safari A, Liu D, Qin J, Songyang Z (2004) The human Rap1 protein complex and modulation of telomere length. The Journal of biological chemistry 279:28585-28591

Omura N, Li CP, Li A, Hong SM, Walter K, Jimeno A, Hidalgo M, Goggins M (2008) Genome-wide profiling of methylated promoters in pancreatic adenocarcinoma. Cancer Biol Ther 7:1146-1156

Palm W, de Lange T (2008) How shelterin protects mammalian telomeres. Annu Rev Genet 42:301-334

Pan H, Guan D, Liu X, Li J, Wang L, Wu J, Zhou J, Zhang W, Ren R, Zhang W et al (2016) SIRT6 safeguards human mesenchymal stem cells from oxidative stress by coactivating NRF2. Cell Res 26:190-205

Proctor CJ, Kirkwood TB (2003) Modelling cellular senescence as a result of telomere state. Aging cell 2:151-157

Reddel RR (2014) Telomere maintenance mechanisms in cancer: clinical implications. Curr Pharm Des 20:6361-6374

Ren R, Deng L, Xue Y, Suzuki K, Zhang W, Yu Y, Wu J, Sun L, Gong $X$, Luan $H$ et al (2017a) Visualization of aging-associated chromatin alterations with an engineered TALE system. Cell Res 27:483-504

Ren R, Ocampo A, Liu GH, Izpisua Belmonte JC (2017b) Regulation of stem cell aging by metabolism and epigenetics. Cell Metab 26:460-474

Sato N, Fukushima N, Chang R, Matsubayashi H, Goggins M (2006) Differential and epigenetic gene expression profiling identifies frequent disruption of the RELN pathway in pancreatic cancers. Gastroenterology 130:548-565
Schmutz I, de Lange T (2016) Shelterin. Curr Biol 26:R397-399

Schulze M, Violonchi C, Swoboda S, Welz T, Kerkhoff E, Hoja S, Bruggemann S, Simburger J, Reinders J, Riemenschneider MJ (2017) RELN signaling modulates glioblastoma growth and substrate-dependent migration. Brain Pathol 28(5):695-709

Sekine K, Kubo K, Nakajima K (2014) How does Reelin control neuronal migration and layer formation in the developing mammalian neocortex? Neurosci Res 86:50-58

Sfeir A, Kabir S, van Overbeek M, Celli GB, de Lange T (2010) Loss of Rap1 induces telomere recombination in the absence of NHEJ or a DNA damage signal. Science 327:1657-1661

Shore D, Nasmyth K (1987) Purification and cloning of a DNA binding protein from yeast that binds to both silencer and activator elements. Cell 51:721-732

Smogorzewska A, de Lange T (2004) Regulation of telomerase by telomeric proteins. Annu Rev Biochem 73:177-208

Takahashi K, Tanabe K, Ohnuki M, Narita M, Ichisaka T, Tomoda K, Yamanaka S (2007) Induction of pluripotent stem cells from adult human fibroblasts by defined factors. Cell 131:861-872

Vincent A, Omura N, Hong SM, Jaffe A, Eshleman J, Goggins M (2011) Genome-wide analysis of promoter methylation associated with gene expression profile in pancreatic adenocarcinoma. Clin Cancer Res 17:4341-4354

Wagner KD, Ying Y, Leong W, Jiang J, Hu X, Chen Y, Michiels JF, Lu Y, Gilson E, Wagner N et al (2017) The differential spatiotemporal expression pattern of shelterin genes throughout lifespan. Aging 9:1219-1232

Wang P, Liu Z, Zhang X, Li J, Sun L, Ju Z, Li J, Chan P, Liu GH, Zhang $W$ et al (2018a) CRISPR/Cas9-mediated gene knockout reveals a guardian role of NF-kappaB/RelA in maintaining the homeostasis of human vascular cells. Protein Cell 9(11):945-965

Wang S, Hu B, Ding Z, Dang Y, Wu J, Li D, Liu X, Xiao B, Zhang W, Ren $R$ et al (2018b) ATF6 safeguards organelle homeostasis and cellular aging in human mesenchymal stem cells. Cell Discov 4:2

Wu Z, Zhang W, Song M, Wang W, Wei G, Li W, Lei J, Huang Y, Sang $Y$, Chan $P$ et al (2018) Differential stem cell aging kinetics in Hutchinson-Gilford progeria syndrome and Werner syndrome. Protein Cell 9:333-350

Xiao L, Lan X, Shi X, Zhao K, Wang D, Wang X, Li F, Huang H, Liu J (2017) Cytoplasmic RAP1 mediates cisplatin resistance of nonsmall cell lung cancer. Cell Death Dis 8:e2803

Xin H, Liu D, Songyang Z (2008) The telosome/shelterin complex and its functions. Genome Biol 9:232

Xu Z, Duc KD, Holcman D, Teixeira MT (2013) The length of the shortest telomere as the major determinant of the onset of replicative senescence. Genetics 194:847-857

Yang D, Xiong Y, Kim H, He Q, Li Y, Chen R, Songyang Z (2011) Human telomeric proteins occupy selective interstitial sites. Cell Res 21:1013-1027

Yang J, Li J, Suzuki K, Liu X, Wu J, Zhang W, Ren R, Zhang W, Chan P, Izpisua Belmonte JC et al (2017) Genetic enhancement in cultured human adult stem cells conferred by a single nucleotide recoding. Cell Res 27:1178-1181 
Yang X, Figueiredo LM, Espinal A, Okubo E, Li B (2009) RAP1 is essential for silencing telomeric variant surface glycoprotein genes in Trypanosoma brucei. Cell 137:99-109

Yu J, Vodyanik MA, Smuga-Otto K, Antosiewicz-Bourget J, Frane JL, Tian S, Nie J, Jonsdottir GA, Ruotti V, Stewart R et al (2007) Induced pluripotent stem cell lines derived from human somatic cells. Science 318:1917-1920

Zha Y, Gan P, Yao Q, Ran FM, Tan J (2014) Downregulation of Rap1 promotes 5-fluorouracil-induced apoptosis in hepatocellular carcinoma cell line HepG2. Oncol Rep 31:1691-1698
Zhang W, Li J, Suzuki K, Qu J, Wang P, Zhou J, Liu X, Ren R, Xu X, Ocampo A et al (2015) Aging stem cells. A Werner syndrome stem cell model unveils heterochromatin alterations as a driver of human aging. Science 348:1160-1163

Zhang W, Wan H, Feng G, Qu J, Wang J, Jing Y, Ren R, Liu Z, Zhang $\mathrm{L}$, Chen $\mathrm{Z}$ et al (2018) SIRT6 deficiency results in developmental retardation in cynomolgus monkeys. Nature 560:661-665

Zhu Y, Liu X, Ding X, Wang F, Geng X (2018) Telomere and its role in the aging pathways: telomere shortening, cell senescence and mitochondria dysfunction. Biogerontology 20:1-16 